\title{
Heavy-particle induced secondary electrons in capacitive radio frequency discharges driven by tailored voltage waveforms
}

\author{
Cite as: J. Appl. Phys. 126, 043303 (2019); doi: 10.1063/1.5100508 \\ Submitted: 17 April 2019 - Accepted: 27 June 2019 . \\ Published Online: 29 July 2019
}

\begin{abstract}
A. Derzsi, ${ }^{1,2, a)}$ (iD B. Horváth, ${ }^{2}$ (D) I. Korolov, ${ }^{3}$ (iD Z. Donkó, ${ }^{2}$ (D) and J. Schulze ${ }^{1,3}$ (D)
\author{
AFFILIATIONS \\ ${ }^{1}$ Department of Physics, West Virginia University, Morgantown, West Virginia 26506, USA \\ ${ }^{2}$ Institute for Solid State Physics and Optics, Wigner Research Centre for Physics, Hungarian Academy of Sciences, \\ 1121 Budapest, Hungary
}

${ }^{3}$ Institute of Electrical Engineering and Plasma Technology, Ruhr-University Bochum, 44780 Bochum, Germany

a) derzsi.aranka@wigner.mta.hu
\end{abstract}

\begin{abstract}
Particle-in-Cell/Monte Carlo Collision simulations are performed to investigate the effects of heavy-particle induced secondary electrons (SEs) on the ionization dynamics and on the control of ion properties at the electrodes in geometrically symmetric capacitively coupled argon discharges driven by tailored voltage waveforms. The driving voltage waveform is composed of a maximum of four $(1 \leq N \leq 4)$ consecutive harmonics of the fundamental frequency of $13.56 \mathrm{MHz}$ and is tailored by adjusting the identical phases of the even harmonics, $\theta$. The simulations are carried out at neutral gas pressures of $3 \mathrm{~Pa}$ (nearly collisionless low-pressure regime) and $100 \mathrm{~Pa}$ (collisional high-pressure regime). Different approaches are used in the simulations to describe the secondary electron emission (SEE) at the electrodes: we adopt (i) constant ion-induced secondary electron emission coefficients (SEECs), $\gamma$, and (ii) realistic, energy-dependent SE yields for ions and fast neutrals. The mean ion energy at the electrodes, $\left\langle E_{\mathrm{i}}\right\rangle$, can be controlled by $\theta$ at both pressures, for both approaches adopted to describe the SEE in the simulations. At a low pressure of $3 \mathrm{~Pa}$, we obtain largely different dependencies of the ion flux at the electrodes, $\Gamma_{\mathrm{i}}$, on $\theta$, depending on the value of the $\gamma$-coefficient. For $\gamma=0.2, \Gamma_{\mathrm{i}}$ remains nearly constant as a function of $\theta$, independently of the choice of $N$, i.e., the mean ion energy can be controlled separately from the ion flux by adjusting $\theta$. However, for values of $\gamma$ different from 0.2 , the quality of the separate control of the ion properties changes significantly. At a high pressure of $100 \mathrm{~Pa}$, independently of the choice of $\gamma$, for a given $N \geq 2$, the ion flux varies as a function of $\theta$. At both pressures, the surface conditions affect the plasma parameters and the quality of the separate control of ion properties at the electrodes. Adopting realistic, energy-dependent SE yields for heavy particles in the simulations can lead to significantly different results compared to those obtained by assuming constant SEECs.
\end{abstract}

Published under license by AIP Publishing. https://doi.org/10.1063/1.5100508

\section{INTRODUCTION}

Low-pressure capacitively coupled plasmas (CCPs) are basic tools in a variety of plasma processing applications. ${ }^{1-3}$ In such applications, which are based on the interactions of plasma particles with the boundary surfaces, the control of particle properties at the surfaces is of key importance. The application of tailored voltage waveforms (TVWs) to generate CCPs offers advanced control of various particle flux-energy distribution functions in technological plasmas. Such waveforms have a nonsinusoidal shape and can be generated as a sum of sinusoidal signals with a fundamental frequency and a number of its higher harmonics with defined phase shifts between them. The waveform shape can be changed by individually varying the harmonics' amplitudes and phases. This affects the sheath voltages at both electrodes and, therefore, the energy of ions arriving at the two electrodes. This type of excitation allows the ion energy to be tuned by the phase angles between the driving harmonics due to the varying dc self-bias that is caused by the electrically asymmetric excitation waveform, i.e., the electrical asymmetry effect (EAE). ${ }^{4-11}$

Particle-in-Cell simulations combined with Monte Carlo type treatment of the collision processes (PIC/MCC method) ${ }^{12-19}$ are 
widely used to study the electron power absorption and ionization dynamics, the discharge characteristics, and the possibilities to control the particle flux-energy distribution functions relevant for plasma processing applications in low-pressure CCPs, including discharges excited by multiple driving frequencies. Such simulations usually adopt several assumptions related to the description of the interaction of plasma particles with the boundary surfaces. Surface coefficients, describing, e.g., the secondary electron emission (SEE), are required as input parameters, which are often unavailable in the literature for different combinations of the discharge gas and the electrode material (with different surface properties) under various discharge conditions. However, the assumptions made on the surface processes, implemented in PIC/MCC simulations of CCPs, have a strong effect on the calculated discharge characteristics. ${ }^{20-27}$

In previous simulation studies of CCPs, the ion-induced secondary electron emission coefficient (SEEC), $\gamma$, was found to affect the electron power absorption and ionization dynamics, i.e., the discharge operation mode. Increasing the value of the $\gamma$-coefficient (which was assumed not to depend on the particle energies, surface properties, and the discharge conditions) was found to result in a transition of the discharge operation mode from the $\alpha$-mode ${ }^{28-34}$ to the $\gamma$-mode. ${ }^{28,35-40}$ In the former case, ionization by electrons accelerated by the expanding sheaths dominates, while in the latter case ionization due to secondary electrons (SEs) in the sheaths is the dominant source of ionization. The value of the $\gamma$-coefficient used in the simulations was found to largely influence the calculated discharge characteristics as well as the control of ion properties at the electrodes in dual-frequency CCPs. ${ }^{35,36,41-43}$ Due to these effects, the importance of the realistic description of the SEE process in kinetic simulations of CCPs was already emphasized..$^{20,26,44,45}$

In order to describe the SEE realistically in PIC/MCC studies of CCPs, the contributions of various plasma particles [ions, fast neutrals, metastables, and vacuum ultraviolet (VUV) photons] to the SEE have to be described on the basis of the energy-dependent secondary yields of all these different particles. ${ }^{46}$ This approach allows to derive so-called "apparent" or "effective" $\gamma^{\star}$ SEECs. ${ }^{47,48}$ Alternatively, SEECs determined in situ by computationally assisted spectroscopic techniques ${ }^{50}$ or SEECs obtained based on theoretical models of the $\mathrm{SEE}^{51}$ can be used in the simulations of CCPs.

The effects of adopting realistic energy-dependent heavyparticle induced SEECs and taking into account the properties of the electrode surfaces on the control of ion properties at the electrodes have previously been studied by PIC/MCC simulations in "classical" dual-frequency discharges (i.e., when two, significantly different driving frequencies are applied). ${ }^{44}$ Simulations in argon were performed by assuming "clean" and "dirty" metal electrodes ${ }^{46}$ in the model, and the effective SEECs, $\gamma^{\star}$, corresponding to these surface conditions were calculated self-consistently. ${ }^{44}$ It was found that $\gamma^{*}$ changes significantly by changing the control parameter for the mean ion energy (the low-frequency voltage amplitude), which negatively influences the quality of the separate control of ion properties at the electrodes. The effects of describing the SEE realistically in PIC/MCC simulations of multifrequency CCPs driven by TVWs on the discharge characteristics have not been investigated so far. In previous studies, (different) constant values for the ioninduced SEEC, $\gamma$, were assumed in electrically asymmetric dualfrequency discharges ${ }^{36}$ and multifrequency discharges. ${ }^{51}$
Here, PIC/MCC simulations are performed at argon gas pressures of $3 \mathrm{~Pa}$ (nearly collisionless low-pressure regime) with a total driving voltage amplitude of $\phi_{\text {tot }}=800 \mathrm{~V}$ and at $100 \mathrm{~Pa}$ (collisional high-pressure regime) with $\phi_{\text {tot }}=120 \mathrm{~V}$. At both pressures, a maximum number of four consecutive harmonics are used to excite the discharge. The phases of the even harmonics are varied from $0^{\circ}$ to $180^{\circ}$, while the phases of the odd harmonics are set to $0^{\circ}$. These conditions are the same as those covered in our previous study in Ref. 51. However, in Ref. 51, only constant ioninduced SEECs were used in the simulations $(\gamma=0.2$ at $3 \mathrm{~Pa}$ and $\gamma=0.4$ at $100 \mathrm{~Pa}$ ). Here, at both pressures, different (constant) values of $\gamma$ between 0.0 and 0.4 are used, and effective heavy-particle induced SEECs, $\gamma^{*}$, are calculated self-consistently for "dirty" and "clean" surface conditions. This way, a more complete picture on the effects of SEs on the discharge characteristics and on the quality of the separate control of the ion properties at the electrodes in multifrequency discharges driven by TVWs is obtained, and the conclusions drawn in Ref. 51 are amended.

The paper is structured in the following way: in Sec. II, we describe the discharge model used in the PIC/MCC simulations and introduce the studied discharge conditions. The simulation results are presented and discussed in Sec. III. The conclusions are drawn in Sec. IV.

\section{SIMULATION METHOD AND DISCHARGE CONDITIONS}

The simulations are based on our one-dimensional in space and three-dimensional in velocity space $(1 \mathrm{~d} 3 \mathrm{v})$ bounded plasma Particle-in-Cell code complemented with Monte Carlo treatment of collision processes (PIC/MCC). ${ }^{18,19}$ The PIC method is widely used for the kinetic description of CCPs. In the frame of this method, "superparticles" (which represent a large number of real plasma particles) are traced and their interactions are considered via a computational mesh. This way the number of particles traced in the simulations can be reduced to a tractable order of magnitude and the immense computational efforts required by the description of their pairwise interactions can be avoided. In the simulations, the particle trajectories (between collisions) are determined based on the solution of the equation of motion and the collisions are described in a stochastic manner (i.e., by the Monte Carlo approach that is based on random numbers which have specific probability distributions according to physical principles).

In the present study, the discharge gas is argon. The particles included in the discharge model are electrons, $\mathrm{Ar}^{+}$ions, and fast Ar neutrals $\left(\mathrm{Ar}^{\mathrm{f}}\right.$, created in charge exchange collisions between $\mathrm{Ar}^{+}$ions and atoms of the background gas, with kinetic energy above the threshold value of $23 \mathrm{eV}$-this energy value is close to the threshold energy for the excitation of Ar atoms by fast neutrals). The ions are traced in the discharge gap until they arrive at the electrodes. The tracing of electrons is executed in a similar way as for ions, except that these particles can be reflected from the surfaces. The fast neutrals are traced until their energy drops below the threshold energy value of $23 \mathrm{eV}$ or until they reach one of the electrodes. The cross sections for the elementary collision processes included in the model are taken from Refs. 52-54. A detailed description of the model can be found in Ref. 20. 
The electrode gap is $L=3 \mathrm{~cm}$ for all conditions investigated here and we consider geometrically symmetric discharges with plane, parallel, and infinite electrodes. One of the electrodes is grounded, while the other one is driven by the following multifrequency voltage waveform, composed of $N$ harmonics of the fundamental frequency, $f=13.56 \mathrm{MHz}$ :

$$
\phi(t)=\sum_{k=1}^{N} \phi_{k} \cos \left(2 \pi k f t+\theta_{k}\right)
$$

with

$$
\phi_{\mathrm{tot}}=\sum_{k=1}^{N} \phi_{k}
$$

In the above equations, $\phi_{k}$ is the amplitude and $\theta_{k}$ is the fixed, but adjustable phase shift of the $k$-th individual harmonic $(k=1, \ldots, N)$, and $\phi_{\text {tot }}$ is the total applied voltage amplitude. The amplitudes of the individual harmonics are set in a way to maximize the electrical generation of the dc self-bias, ${ }^{55}$

$$
\phi_{k}=\phi_{0} \frac{N-k+1}{N},
$$

where

$$
\phi_{0}=\frac{2 \phi_{\mathrm{tot}}}{N+1} .
$$

So-called "peaks"-type voltage waveforms can be generated by setting all $\theta_{k}$ phase angles to zero in Eq. (1), while "valleys"type waveforms can be obtained by changing the phase angles of all the even harmonics to $180^{\circ}$. In Fig. 1, examples of the normalized driving voltage waveform, $\phi(t) / \phi_{\text {tot }}$, are shown for specific values of $N$ and $\theta_{k}$.
In PIC/MCC simulations of CCPs, the interactions of various plasma particles with the boundary surfaces have to be described. This can be done by adopting simple approaches (by defining constant surface coefficients for the different surface processes as input parameters to the simulation) or by realistic approaches (where the surface properties as well as the dependence of the surface processes on the energy of the particles are taken into account). Here, we assume that the electrodes are made of the same (metal) material with identical surface conditions. The electron reflection probability is set to 0.2 for all conditions (simple approach), ${ }^{56}$ independently of the electrons' energy and their angle of incidence. Other types of electron-surface processes, e.g., the emission of SEs due to electron impact, are neglected. We note that recent studies have found considerable effects of the electron induced SEs on the plasma parameters at specific discharge conditions ${ }^{57,58}$, i.e., at very low pressures $(p \ll 3 \mathrm{~Pa})$ and high voltage amplitudes, for dielectric surfaces. However, in the present study, metal electrodes are assumed (compared to dielectric surfaces, the maximum of the electron induced SE yield is much lower for metal surfaces), and the discharge conditions covered here are significantly different (higher pressures, lower $\phi_{\text {tot }}$ ) compared to those in Refs. 57 and 58.

SEE induced by heavy particles ( $\mathrm{Ar}^{+}$ions and $\mathrm{Ar}^{\mathrm{f}}$ neutrals) is taken into account in the simulations. Both simple and realistic approaches are used to describe the SEE processes induced by these particles at the electrodes: (i) constant ion-induced SEECs, $\gamma$, taking values between 0.0 and 0.4 are used (simple approach) and (ii) the $\mathrm{SE}$ yield of the individual $\mathrm{Ar}^{+}$ions and $\mathrm{Ar}^{\mathrm{f}}$ neutrals hitting the electrodes, $\gamma_{\mathrm{i}}$ and $\gamma_{\mathrm{a}}$, respectively, are considered as a function of the incident particle energy (realistic approach). For argon ions and neutrals, $\gamma_{i}$ and $\gamma_{a}$ are given in Refs. 46 and 59 for "dirty" metal electrodes as well as "clean" surface conditions. "Clean" electrodes represent atomically clean surface conditions (e.g., sputtered surfaces in the high vacuum environment), while "dirty" electrodes represent surfaces under typical laboratory conditions (treated by the standard

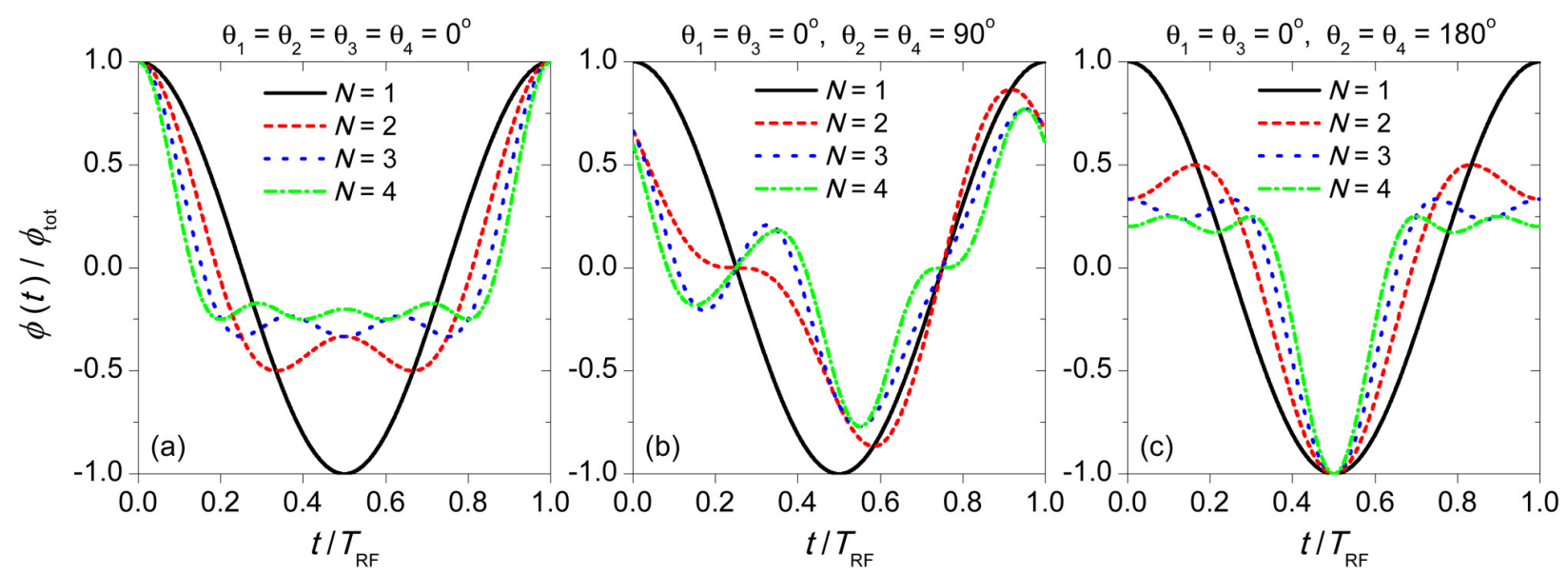

FIG. 1. Normalized driving voltage waveforms for exemplary choices of $N$ and $\theta_{k}$, shown for one period of the fundamental frequency $\left(T_{\mathrm{RF}}=1 / f\right)$. 
chemical and mechanical cleaning procedures, contaminated or oxidized samples). ${ }^{46}$ Here, simulations are run by assuming both "dirty" and "clean" surface conditions.

For "dirty" electrodes, the following formulas, given in Ref. 46 and corrected in Ref. 59, are used to compute the SE yield of the individual ions $\left(\gamma_{\mathrm{i}}\right)$ and fast neutrals $\left(\gamma_{\mathrm{a}}\right)$ hitting the electrodes as a function of their energy, $\epsilon$ (given in electron volt),

$$
\begin{gathered}
\gamma_{\mathrm{i}}(\epsilon)=\frac{0.006 \epsilon}{1+(\epsilon / 10)}+\frac{1.05 \times 10^{-4}(\epsilon-80)^{1.2}}{1+(\epsilon / 8000)^{1.5}}, \\
\gamma_{\mathrm{a}}(\epsilon)=\frac{1 \times 10^{-4}(\epsilon-90)^{1.2}}{1+(\epsilon / 8000)^{1.5}}+\frac{7 \times 10^{-5}(\epsilon-32)^{1.2}}{1+(\epsilon / 2800)^{1.5}} .
\end{gathered}
$$

To calculate $\gamma_{\mathrm{i}}$ and $\gamma_{\mathrm{a}}$ for "clean" surfaces, the formulas given in Ref. 46 are used,

$$
\begin{gathered}
\gamma_{\mathrm{i}}(\epsilon)=0.07+1 \times 10^{-5}(\epsilon-500)^{1.2} /\left[1+(\epsilon / 70000)^{0.7}\right], \\
\gamma_{\mathrm{a}}(\epsilon)=1 \times 10^{-5}(\epsilon-500)^{1.2} /\left[1+(\epsilon / 70000)^{0.7}\right] .
\end{gathered}
$$

Based on the energy-dependent SE yields of the individual heavy particles, calculated according to the above formulas (shown also in Fig. 2 as a function of the incident particle energy for different surface conditions), the effective SEEC, $\gamma^{*}$, can be computed as proposed in Refs. 47 and 48,

$$
\gamma^{*}=\frac{\sum_{\mathrm{k}=1}^{\mathrm{N}_{\mathrm{i}}} \gamma_{\mathrm{i}}\left(\epsilon_{\mathrm{k}}\right)+\sum_{\mathrm{k}=1}^{\mathrm{N}_{\mathrm{a}}} \gamma_{\mathrm{a}}\left(\epsilon_{\mathrm{k}}\right)}{\mathrm{N}_{\mathrm{i}}},
$$

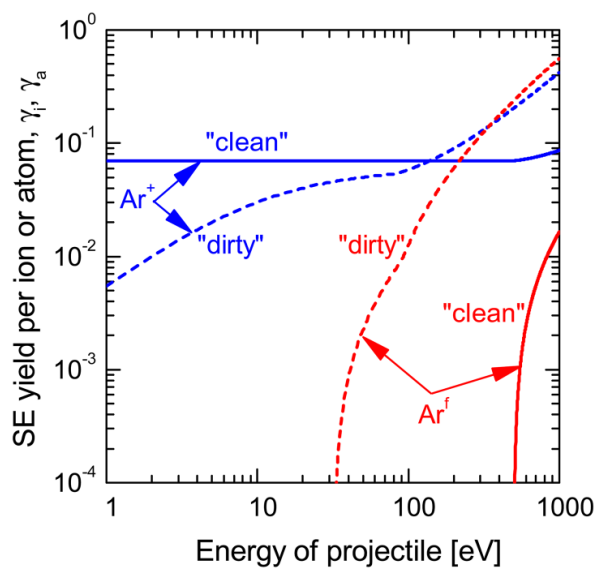

FIG. 2. Secondary electron yields on "dirty" (dashed lines) and "clean" (solid lines) metal surfaces due to $\mathrm{Ar}^{+}$ions and fast $\mathrm{Ar}$ neutrals $\left(\mathrm{Ar}^{\mathrm{f}}\right)$ as a function of the incident particle energy. The secondary electron yields are calculated based on formulas from Refs. 46 and 59. where $\epsilon_{k}$ is the energy of the $k$-th ion or atom traced in the simulation (upon arrival at the electrode) and $N_{\mathrm{i}}$ and $N_{\mathrm{a}}$ are respectively, the total number of $\mathrm{Ar}^{+}$ions and $\mathrm{Ar}^{\mathrm{f}}$ atoms reaching a given electrode during a RF period. Under the conditions investigated here, mainly these plasma species contribute to SEE; therefore, the contributions of other plasma species are neglected in Eq. (9).

In the simulations performed for "dirty" surface conditions, Eqs. (5), (6), and (9) are used to calculate the effective SEEC, $\gamma_{\mathrm{d}}^{*}$. In the simulations performed for "clean" surface conditions, the effective SEEC, $\gamma_{\mathfrak{c}}^{*}$, is obtained based on Eqs. (7)-(9).

The simulations are performed at neutral gas pressures of $3 \mathrm{~Pa}$ (nearly collisionless low-pressure regime) with $\phi_{\text {tot }}=800 \mathrm{~V}$ and at $100 \mathrm{~Pa}$ (collisional high-pressure regime) with $\phi_{\text {tot }}=120 \mathrm{~V}$. For these values of the total voltage amplitudes, convergence of the simulations could be reached for all values of $N, \theta$, and even for the highest values of $\gamma$ assumed in the simulations. A maximum number of four consecutive harmonics are used at both pressures, to excite the discharge $(1 \leq N \leq 4)$. The phases of the even harmonics $\left(\theta_{2}=\theta_{4}=\theta\right)$ are varied from $0^{\circ}$ to $180^{\circ}$, while the phases of the odd harmonics are kept constant at $0^{\circ}\left(\theta_{1}=\theta_{3}=0^{\circ}\right)$.

In the simulations, the background gas density and the gas temperature are constant in space and time. The energy transfer to the background gas atoms, i.e., the heating of the discharge gas, is not calculated directly in the simulations. However, at the studied discharge conditions, the gas temperature is expected to be significantly higher than the room temperature, especially at high total voltage amplitudes. Therefore, a constant neutral gas temperature of $400 \mathrm{~K}$ is assumed in the present study.

In the simulations where constant $\gamma$ coefficients are used, the traced particles are electrons and $\mathrm{Ar}^{+}$ions, while in the simulations where the SE yields are calculated realistically fast neutrals $\left(\operatorname{Ar}^{\mathrm{f}}\right)$ are also traced.

\section{RESULTS}

\section{A. Low-pressure regime $(3 \mathrm{~Pa})$}

In this section, PIC/MCC simulation results obtained for $3 \mathrm{~Pa}$ and $\phi_{\text {tot }}=800 \mathrm{~V}$ are presented. Firstly, results obtained by using constant values for the SEEC, $\gamma$, are discussed. $\gamma=0.0,0.2$, and 0.3 are set in the simulations for $N=1,2$, and $4 . \gamma=0.3$ was the highest possible value for which convergence of the simulations could be achieved in the whole $0^{\circ}-180^{\circ}$ domain of $\theta$ under the discharge conditions studied here. Secondly, the discharge characteristics resulting from simulations with energy-dependent heavy-particle induced SEECs for "dirty" and "clean" surface conditions are presented.

\section{Constant heavy-particle induced SEECs, $\gamma$, at $3 \mathrm{~Pa}$}

In Fig. 3 , the dc self-bias voltage, $\eta$, is shown as a function of the identical phase angle of the even harmonics, $\theta=\theta_{2}=\theta_{4}$, for different numbers of applied harmonics, $N$, and different (constant) values of the $\gamma$-coefficient $(\gamma=0.0,0.2,0.3)$. By changing $\theta$, the $\mathrm{dc}$ self-bias voltage can be tuned efficiently, while adding more consecutive harmonics to the driving voltage waveform, i.e., increasing $N$, enlarges the interval over which $\eta$ can be controlled. At $N=2,|\eta|$ reaches a maximum value of about $330 \mathrm{~V}\left(|\eta| / \phi_{\text {tot }}=41 \%\right)$, while at 


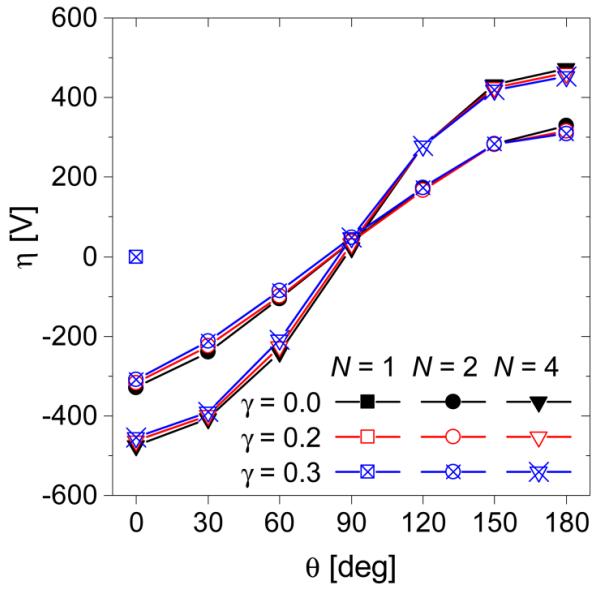

FIG. 3. Dependence of the dc self-bias voltage, $\eta$, on the identical phase angles of the even harmonics, $\theta=\theta_{2}=\theta_{4}\left(\theta_{1}=\theta_{3}=0^{\circ}\right)$, for different numbers of applied harmonics, $N$, and different values of the $\gamma$-coefficient $(\gamma=0.0$, $0.2,0.3)$. Discharge conditions: $p=3 \mathrm{~Pa}, \phi_{\text {tot }}=800 \mathrm{~V}$. Note that the symbols completely overlap in some cases, e.g., at $N=1$.

$N=4$ the dc self-bias voltage can be tuned from about $-475 \mathrm{~V}$ to $475 \mathrm{~V}\left(|\eta| / \phi_{\text {tot }}=59 \%\right)$ by changing $\theta$ from $0^{\circ}$ to $180^{\circ}$. Almost the same values of $\eta$ (max. 5\% difference) as a function of $\theta$ are obtained for all three different values of $\gamma$, i.e., the choice of $\gamma$ does not affect significantly the dc self-bias voltage.

By changing $\eta$ via the phase angles, the mean ion energy at the electrodes can be controlled. This can be seen in panels (a) and (c) of Fig. 4, where the mean ion energies, $\left\langle E_{\mathrm{i}}\right\rangle$, at the grounded and powered electrodes are shown as a function of $\theta$ for different numbers of applied harmonics and different values of $\gamma .\left\langle E_{\mathrm{i}}\right\rangle$ can be increased by a factor of about 7 at $N=4$ at the grounded electrode (from about $50 \mathrm{eV}$ up to about $330 \mathrm{eV}$ ) and decreased at the powered electrode by changing $\theta$ from $0^{\circ}$ to $180^{\circ}$. The mean ion energies at both electrodes are slightly affected by the value of the $\gamma$-coefficient assumed in the simulations. At a given $N$ and $\theta$, the lowest mean ion energies are obtained for $\gamma=0.0$. Compared to these results, by using $\gamma=0.3$ in the simulations, an increase of the mean ion energy by a maximum of $18 \%$ can be observed, at a given $N$ and $\theta$. This can be explained by the effects of SEs on the ionization dynamics, the plasma density, and the sheath length. At higher values of $\gamma$, more SEs are emitted from the electrodes, which are accelerated in the sheaths and induce significant ionization. This leads to an increase of the plasma density and a decrease of the sheath lengths at both electrodes (see the effects of $\gamma$ on the ionization dynamics and the length of the sheaths for $N=4$ and different phase angles in Fig. 5). The narrow and weakly collisional sheaths under low-pressure conditions cause $\left\langle E_{\mathrm{i}}\right\rangle$ to slightly increase at both electrodes by increasing $\gamma$.

In our previous study performed at the same discharge conditions ( $3 \mathrm{~Pa}$ pressure, $3 \mathrm{~cm}$ electrode gap, and total voltage amplitude of $\phi_{\text {tot }}=800 \mathrm{~V}$ ) with $\gamma=0.2$, we found that the mean ion energy can be controlled separately from the ion flux by adjusting $\theta:{ }^{51}$ it was demonstrated that the ion flux, $\Gamma_{\mathrm{i}}$, remains essentially constant as a function of $\theta$, independently of the choice of $N$, at both electrodes, while the mean ion energy can be controlled by $\theta$. This can also be seen in Fig. 4 [the ion fluxes at the grounded and powered electrodes are shown in panels (b) and (d), respectively], where the results obtained for $\gamma=0.2$ are shown as open symbols. Compared to these results, for values of $\gamma$ different from 0.2 the quality of the separate control of the ion properties changes significantly. For $\gamma=0.0$ (filled symbols), $\Gamma_{\mathrm{i}}$ first decreases, then increases by changing $\theta$ from $0^{\circ}$ to $180^{\circ}$. The lowest ion fluxes are found at $\theta=90^{\circ}$, for all $N \geq 2$. At $N=2, \Gamma_{\mathrm{i}}$ decreases by a maximum of $22 \%$, while at $N=4$ a decrease by a maximum of $35 \%$ is found by tuning $\theta$ from $0^{\circ}$ to $90^{\circ}$. Under such conditions, the separate control of $\left\langle E_{\mathrm{i}}\right\rangle$ and $\Gamma_{\mathrm{i}}$ cannot be realized. Similarly, by assuming a high SE yield of $\gamma=0.3$ in the simulations (results shown with open crossed symbols in Fig. 4), $\Gamma_{\mathrm{i}}$ does not remain constant as a function of $\theta$. However, as opposed to the results for $\gamma=0.0$, here the highest ion fluxes are obtained at $\theta=90^{\circ}$, i.e., for all $N \geq 2, \Gamma_{\mathrm{i}}$ first increases, then decreases by changing $\theta$ from $0^{\circ}$ to $180^{\circ}$. At $N=2, \Gamma_{\mathrm{i}}$ increases by a maximum of $55 \%$, while at $N=4$ an increase by a maximum of $70 \%$ is obtained as $\theta$ is increased from $0^{\circ}$ to $90^{\circ}$.

These results show that the quality of the separate control of the ion properties at the electrodes is largely influenced by the emission of SEs in low-pressure discharges excited by multifrequency TVWs. Depending on the value of $\gamma$ assumed in the simulations, largely different dependencies of $\Gamma_{\mathrm{i}}$ on the control parameter for the mean ion energy, $\theta$, are obtained. While at $\gamma=0.2$ the separate control of ion properties seems to be achievable, using lower/higher values for $\gamma$ in the simulations results in varying ion fluxes as a function of $\theta$, i.e., both $\Gamma_{\mathrm{i}}$ and $\left\langle E_{\mathrm{i}}\right\rangle$ change by tuning $\theta$. The decrease/ increase of $\Gamma_{\mathrm{i}}$ by tuning $\theta$ from $0^{\circ}$ to $90^{\circ}$ at low/high values of $\gamma$ ( 0.0 and 0.3 , respectively) is much stronger at $N=4$ compared to $N=2$. The quality of the separate control of ion properties is more affected (reduced) by SEs at higher numbers of applied harmonics. Strong effects of SEs on the quality of the control of ion properties at the electrodes have previously been demonstrated in classical dualfrequency discharges, ${ }^{35,44}$ as well as in electrically asymmetric dualfrequency discharges ${ }^{36}$ at somewhat different discharge conditions.

The influence of SEs on the discharge characteristics, especially the different dependence of the $\Gamma_{\mathrm{i}}$ ion flux on $\theta$ at different values of $\gamma$, can be understood based on an analysis of the ionization dynamics. Figure 5 shows the spatio-temporal plots of the ionization rate for $N=4$ applied harmonics, for different phase angles $\left(\theta=0^{\circ}, 90^{\circ}\right.$, and $\left.180^{\circ}\right)$, and for different values of the $\gamma$-coefficient $(\gamma=0.0,0.2$, and 0.3$)$. In all cases, the discharge operates in the $\alpha$-mode, tuning $\theta$ or varying $\gamma$ does not change the dominant power absorption mode in the discharge. However, the symmetry of the ionization dynamics is influenced by the phase angle, $\theta$, and this affects the ionization induced by SEs. At $\theta=0^{\circ} / \theta=180^{\circ}$, strong $\alpha$-mode ionization, enhanced by nonlinear electron resonance heating (NERH), ${ }^{60-64}$ is found at the powered/grounded electrode, respectively, for all $\gamma$. At $\theta=90^{\circ}$, the spatio-temporal ionization is symmetric, without NERH. At $\gamma=0.0$ (top row in Fig. 5), the ionization maxima at $\theta=90^{\circ}$ are significantly weaker than those obtained for $\theta=0^{\circ}$ and $\theta=180^{\circ}$, and less ionization is produced on time and space average at this phase angle compared to $\theta=0^{\circ}$ 

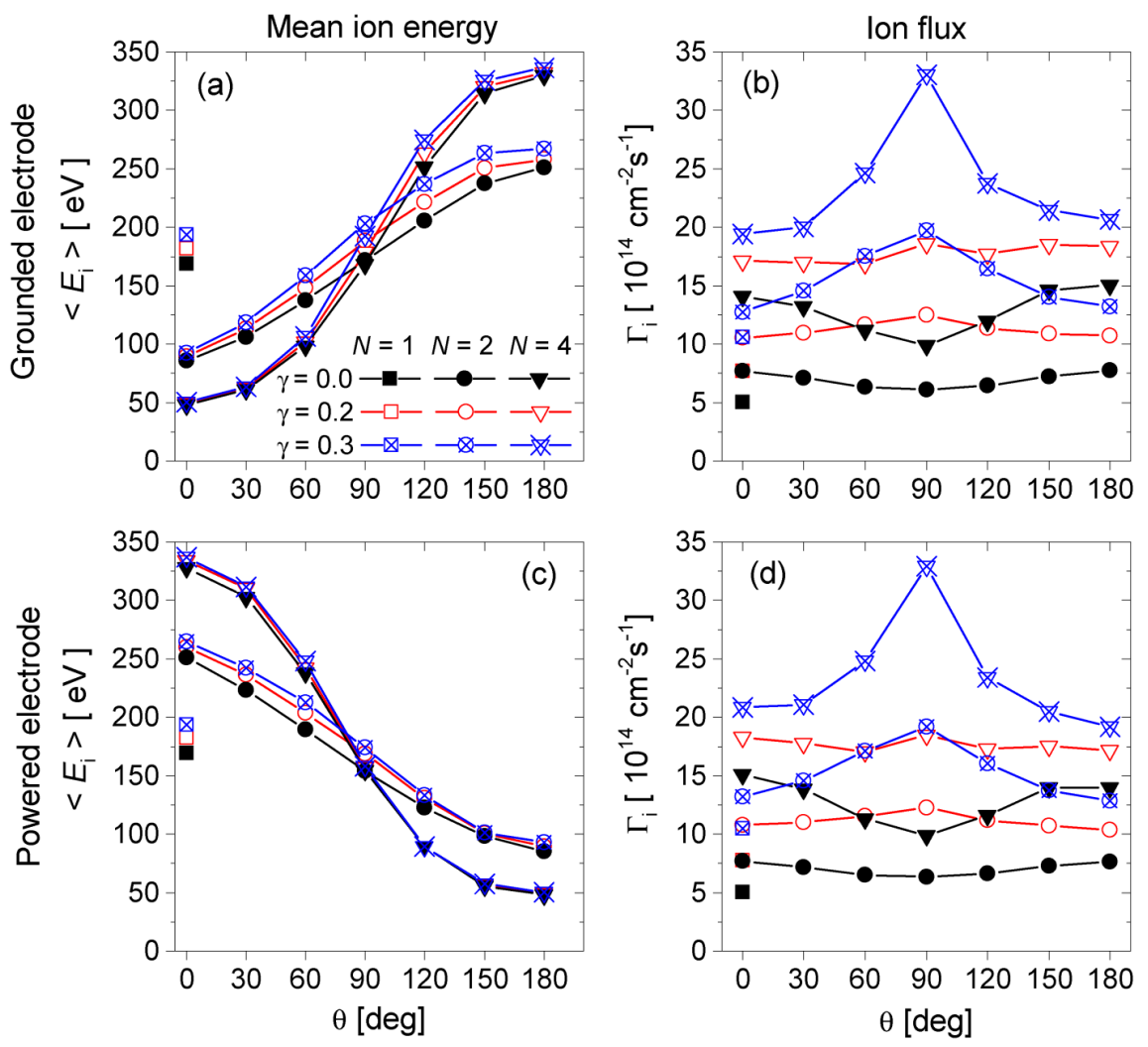

FIG. 4. Mean ion energy (panels on the left), $\left\langle E_{\mathrm{i}}\right\rangle$, and ion flux (panels on the right), $\Gamma_{\mathrm{i}}$, at the grounded and powered electrodes (top and bottom panels, respectively) as a function of $\theta=\theta_{2}=\theta_{4}\left(\theta_{1}=\theta_{3}=0^{\circ}\right)$ for different numbers of applied harmonics, $N$, and different values of the $\gamma$-coefficient $(\gamma=0.0,0.2,0.3)$. Discharge conditions: $p=3 \mathrm{~Pa}, \phi_{\text {tot }}=800 \mathrm{~V}$. Note that the symbols completely overlap in some cases. and $180^{\circ}$. This is caused by the slower sheath expansion velocity at $90^{\circ}$. As a consequence of this, the ion flux reaches its minimum at $\theta=90^{\circ}$, where it is about $35 \%$ lower than those at $0^{\circ}$ and $180^{\circ}$ [Figs. 4(b) and 4(d), $\Gamma_{\mathrm{i}}$ shown with solid triangles for $N=4$ ].

At $\gamma=0.2$ (center row in Fig. 5), the SEs emitted from the electrodes and accelerated in the sheath induce ionization, which enhances the plasma density and leads to an increase of the ion flux at both electrodes, at all $\theta$. Compared to the ion fluxes obtained for $\gamma=0.0$, by assuming $\gamma=0.2$ in the simulations, $\Gamma_{\mathrm{i}}$ increases by a factor of about 1.2 at $\theta=0^{\circ}$ and $\theta=180^{\circ}$. However, at these phase angles, poor confinement of SEs is found. The SEs created at the powered/grounded electrode at $\theta=180^{\circ} / 0^{\circ}$ during the time of sheath expansion at a given electrode are mainly lost at the other (grounded/powered) electrode where the sheath is collapsed for a long fraction of one period of the fundamental frequency, $T_{\mathrm{RF}}$. For values of $\theta$ around $90^{\circ}$, good confinement of SEs is found due to the high sheath voltages at both electrodes during a long fraction of $T_{\mathrm{RF}}$. This leads to an increase of the ion flux at both electrodes. At $\theta=90^{\circ}$, the ion flux is about 1.8 times higher than that at $\gamma=0.0$. At values of $\theta$ around $90^{\circ}$, the ionization induced by SEs compensates the weaker $\alpha$-mode ionization, resulting in ion fluxes similar to those obtained at $\theta=0^{\circ}$ and $180^{\circ}$, where the strong $\alpha$-mode ionization is further enhanced by NERH. Under these conditions, on time and space average, the ionization rates are similar, independently of $\theta$; therefore, the ion fluxes remain nearly constant as a function of $\theta$ at both electrodes.
Compared to the $\gamma=0.2$ case, more SEs are emitted from the electrodes at $\gamma=0.3$, which further enhance the ionization in the discharge (bottom row in Fig. 5). At $\theta=0^{\circ}$ and $180^{\circ}$, this effect results in an additional increase of the ion fluxes by about $15 \%$. This moderate increase of the ion fluxes, besides the poor confinement of SEs at these phase angles, is also related to the longer mean free paths and the less efficient multiplication of SEs at lowpressure conditions. However, at $\theta=90^{\circ}$, the high number of SEs as a result of the high $\gamma$ in combination with the good confinement of SEs results in highly efficient multiplication of SEs in the bulk region (due to their multiple reflections between the sheaths) [Fig. 5(h)] despite the low pressure of $3 \mathrm{~Pa}$ of the discharge gas. As a result, the ion flux increases by about $80 \%$ at $\theta=90^{\circ}$ compared to $\Gamma_{\mathrm{i}}$ obtained for $\gamma=0.2$ at the same phase angle.

The effects of heavy-particle induced SEs on the quality of the separate control of the ion properties at the electrodes (via the control parameter, $\theta$ ) seen here for TVWs are similar to the scenario found previously in classical dual-frequency discharges driven by two significantly different frequencies. ${ }^{35,36}$ In classical dual-frequency discharges (where the control parameter of the mean ion energy is the low-frequency voltage amplitude, while the ion flux is tuned by the high-frequency voltage amplitude), the separate control of the ion properties at the electrodes can be realized only for specific choices of $\gamma$. Low values of $\gamma$ cause a decrease of $\Gamma_{\mathrm{i}}$ and high values of $\gamma$ cause an increase of $\Gamma_{i}$ as a function of the control parameter of the mean ion energy, i.e., the low-frequency voltage amplitude. 

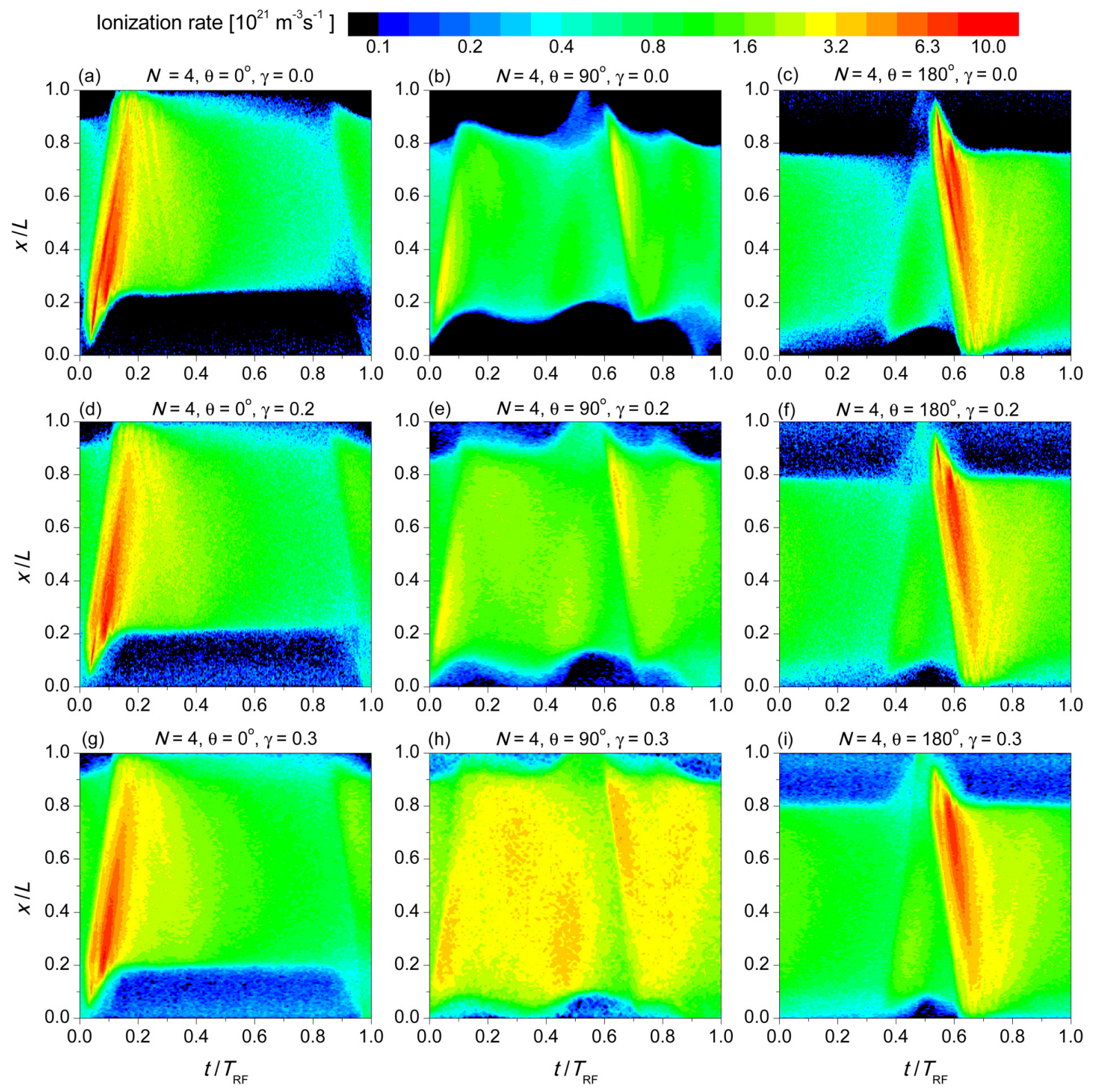

FIG. 5. Spatio-temporal plots of the ionization rate for $N=4$ applied harmonics, for different phase angles: $\theta=0^{\circ}$ (left column), $\theta=90^{\circ}$ (center column), and $\theta=180^{\circ}$ (right column), and for different values of the $\gamma$-coefficient: $\gamma=0.0$ (top row), $\gamma=0.2$ (center row), and $\gamma=0.3$ (bottom row). The powered electrode is at $x / L=0$, while the grounded electrode is at $x / L=1$. $T_{\mathrm{RF}}$ indicates one period of the fundamental frequency. Discharge conditions: $L=3 \mathrm{~cm}, p=3 \mathrm{~Pa}, \phi_{\text {tot }}=800 \mathrm{~V}, \theta=\theta_{2}=\theta_{4}$, $\theta_{1}=\theta_{3}=0^{\circ}$. The color scale is logarithmic.

\section{Realistic heavy-particle induced SEECs, $\gamma^{*}$, at $3 \mathrm{~Pa}$}

In the following, the results of simulations in which the SE yields, as a result of heavy particle bombardment of the electrodes, were calculated as a function of the particle energy, are presented. The discharge conditions are the same as above $(3 \mathrm{~cm}$ electrode gap, $3 \mathrm{~Pa}$, and $\phi_{\mathrm{tot}}=800 \mathrm{~V}$ ). Both "dirty" and "clean" surface conditions are covered in this study. The effective SEECs for these surface conditions, $\gamma_{\mathrm{d}}^{*}$ and $\gamma_{\mathrm{c}}^{*}$, are calculated as described in Sec. II.

Figure 6 shows the dc self-bias voltage, $\eta$, as a function of the identical phase angles of the even harmonics, $\theta=\theta_{2}=\theta_{4}$, for different numbers of applied harmonics, $N$, for "dirty" and "clean" 


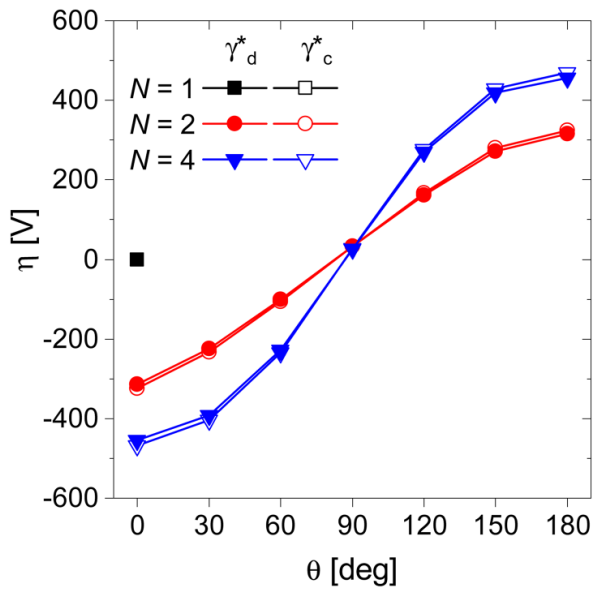

FIG. 6. Dependence of the dc self-bias voltage, $\eta$, on the identical phase angle of the even harmonics, $\theta=\theta_{2}=\theta_{4}\left(\theta_{1}=\theta_{3}=0^{\circ}\right)$, for different numbers of applied harmonics, $N$, obtained from simulations where the effective SEECs for "dirty" (filled symbols) and "clean" (open symbols) surface conditions, $\gamma_{d}^{*}$ and $\gamma_{\mathrm{c}}^{*}$, respectively, are calculated self-consistently. Discharge conditions: $p=3$ $\mathrm{Pa}, \phi_{\text {tot }}=800 \mathrm{~V}$. Note that the symbols completely overlap in some cases. surface conditions. Similar to the results shown in Fig. 3 for constant ion-induced SEECs, by changing $\theta$ the dc self-bias voltage can be tuned efficiently. There is no significant difference between the results obtained for "dirty" and "clean" surfaces (the surface conditions do not affect significantly the dc self-bias voltage under these conditions, the differences are always below $3 \%$ at a given $N$ and $\theta$ ), and the $|\eta|$ values at both $N=2$ and $N=4$ are close to those obtained by assuming constant $\gamma$-coefficients (Fig. 6 compared to Fig. 3): at $N=2, \eta$ can be tuned within about $\pm 315 \mathrm{~V}$, while at $N=4$ it can be changed within about $\pm 455 \mathrm{~V}$ by tuning $\theta$ from $0^{\circ}$ to $180^{\circ}$.

Figure 7 shows the mean ion energy, $\left\langle E_{\mathrm{i}}\right\rangle$, and the ion flux, $\Gamma_{\mathrm{i}}$, at the grounded and powered electrodes as a function of $\theta=\theta_{2}=\theta_{4}$ for different $N$, for "dirty" (filled symbols) and "clean" (open symbols) surface conditions. By changing $\theta$, the mean ion energy at the electrodes can be controlled [panels (a) and (c)], similar to the results of simulations with constant $\gamma$-coefficients. For both surface conditions, $\left\langle E_{\mathrm{i}}\right\rangle$ can be increased/decreased by a factor of about 2.5 at $N=2$ and by a factor of about 7 at $N=4$ at the grounded/ powered electrode by changing $\theta$ from $0^{\circ}$ to $180^{\circ}$. While the mean ion energies are almost the same for both "dirty" and "clean" surface conditions at a given $N$ and $\theta$, the ion fluxes are affected by the surface conditions at both electrodes, as it can be seen in panels (b)
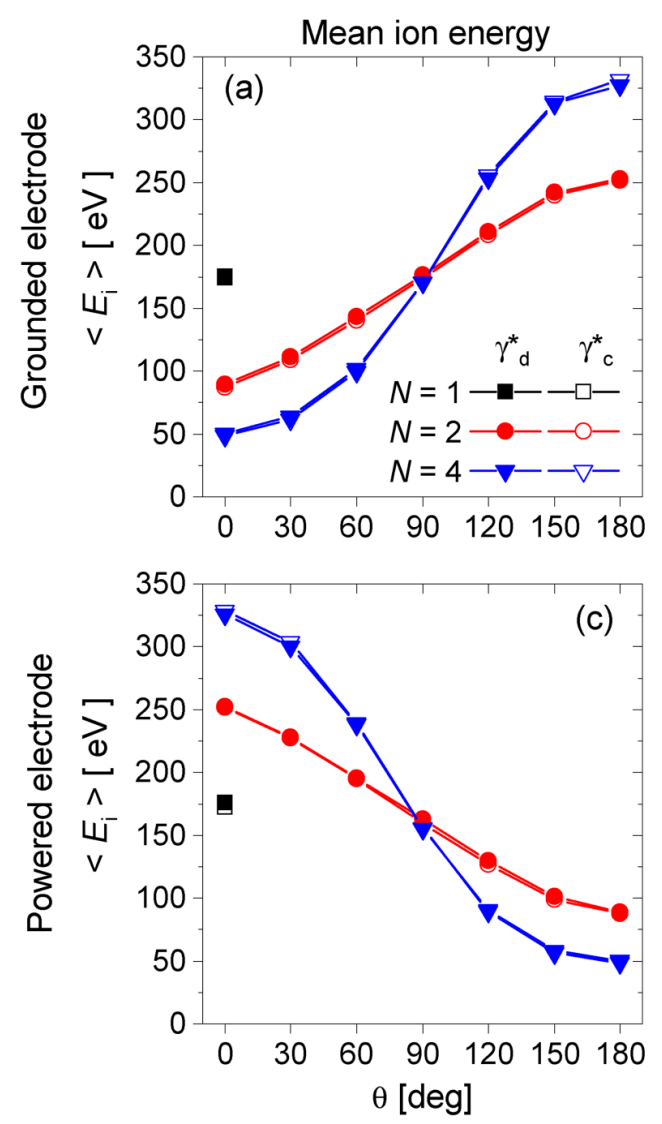
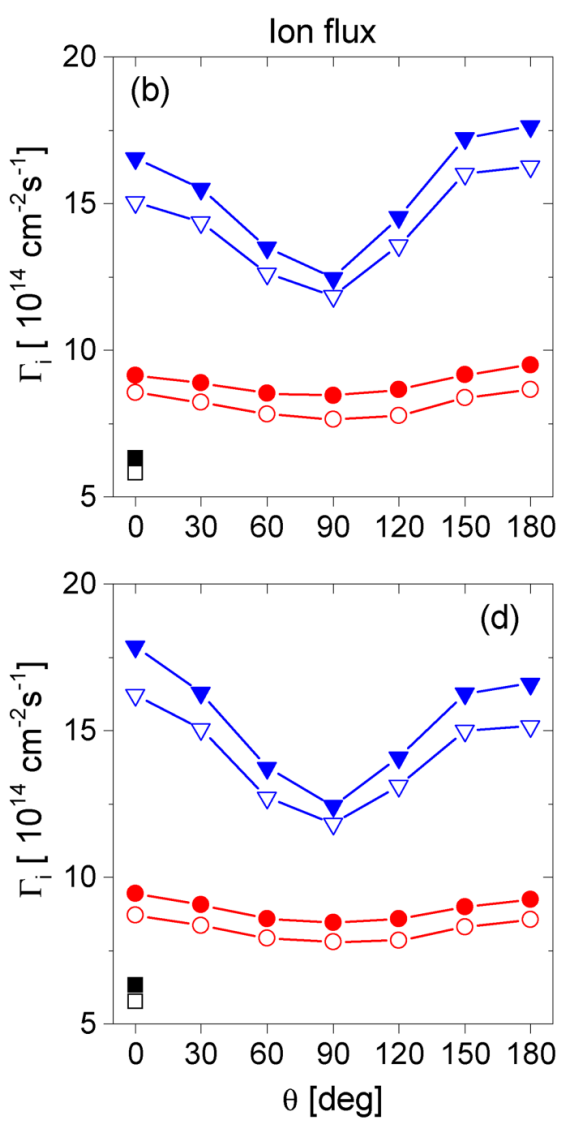

FIG. 7. Mean ion energy (panels on the left), $\left\langle E_{\mathrm{i}}\right\rangle$, and ion flux (panels on the right), $\Gamma_{\mathrm{i}}$, at the grounded and powered electrodes (top and bottom panels, respectively) as a function of $\theta=\theta_{2}=\theta_{4}\left(\theta_{1}=\theta_{3}=0^{\circ}\right)$ for different numbers of applied harmonics, $N$, obtained from simulations with selfconsistently calculated effective SEECs, $\gamma_{d}^{*}$ and $\gamma_{c}^{*}$, for "dirty" (filled symbols) and "clean" (open symbols) surface conditions, respectively. Discharge conditions: $p=3 \mathrm{~Pa}, \phi_{\text {tot }}=800 \mathrm{~V}$. Note that the symbols completely overlap in some cases. 


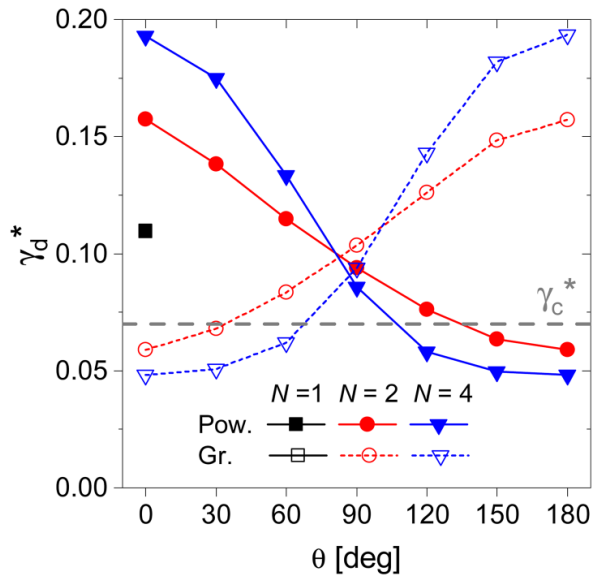

FIG. 8. Self-consistently calculated effective SEEC for "dirty" surface conditions, $\gamma_{\mathrm{d}}^{*}$, at the powered and grounded electrodes (filled and open symbols, respectively) as a function of $\theta=\theta_{2}=\theta_{4}\left(\theta_{1}=\theta_{3}=0^{\circ}\right)$ for different numbers of applied harmonics, $N$. The dashed line shows the value of the effective SEEC for "clean" surface conditions, $\gamma_{c}^{*}$, which is 0.07 for all $\theta$ and $N$ at both electrodes. Discharge conditions: $p=3 \mathrm{~Pa}, \phi_{\mathrm{tot}}=800 \mathrm{~V}$. Note that the symbols completely overlap at $N=1$. and (d) of Fig. 7. For "dirty" surfaces, higher (up to 10\%) ion fluxes are obtained at all $\theta$ and $N$, and the difference between the fluxes at different surface conditions slightly increases with increasing number of applied harmonics.

At $N=2, \Gamma_{\mathrm{i}}$ changes by up to $12 \%$ by tuning $\theta$ from $0^{\circ}$ to $180^{\circ}$ for both "dirty" and "clean" surfaces, with the lowest ion fluxes obtained at $\theta=90^{\circ}$. At $N=4$, the ion flux changes by up to about $45 \%$ for "dirty" surfaces and up to about $37 \%$ for "clean" surfaces, by changing $\theta$ from $0^{\circ}$ to $180^{\circ}$. These simulations show that by describing the SEE realistically in the simulations via a selfconsistent calculation of the SEECs, $\gamma_{d}^{*}$ and $\gamma_{c}^{*}$, a different dependence of $\Gamma_{\mathrm{i}}$ on $\theta$ is obtained compared to those obtained for constant $\gamma$-coefficients [see Figs. 7(b) and 7(d) compared to Figs. 4(b) and $4(\mathrm{~d})]$. Here, at both surface conditions, $\Gamma_{\mathrm{i}}$ exhibits maxima at $\theta=0^{\circ}$ and $180^{\circ}$, and a minimum value at $\theta=90^{\circ}$, independently of $N$. This behavior of the ion flux as a function of $\theta$ can be understood based on the analysis of the self-consistently calculated effective SEECs shown in Fig. 8, the flux-energy distributions of ions and fast neutrals at the electrodes (Fig. 9), and the spatiotemporal ionization dynamics (Fig. 10).

For "dirty" surface conditions the self-consistently calculated effective SEEC, $\gamma_{\mathrm{d}}^{*}$, changes as a function of $\theta$ at both electrodes (Fig. 8). By tuning $\theta$ from $0^{\circ}$ to $180^{\circ}, \gamma_{\mathrm{d}}^{*}$ increases at the grounded electrode (from about 0.06 to about 0.16 at $N=2$, and from about
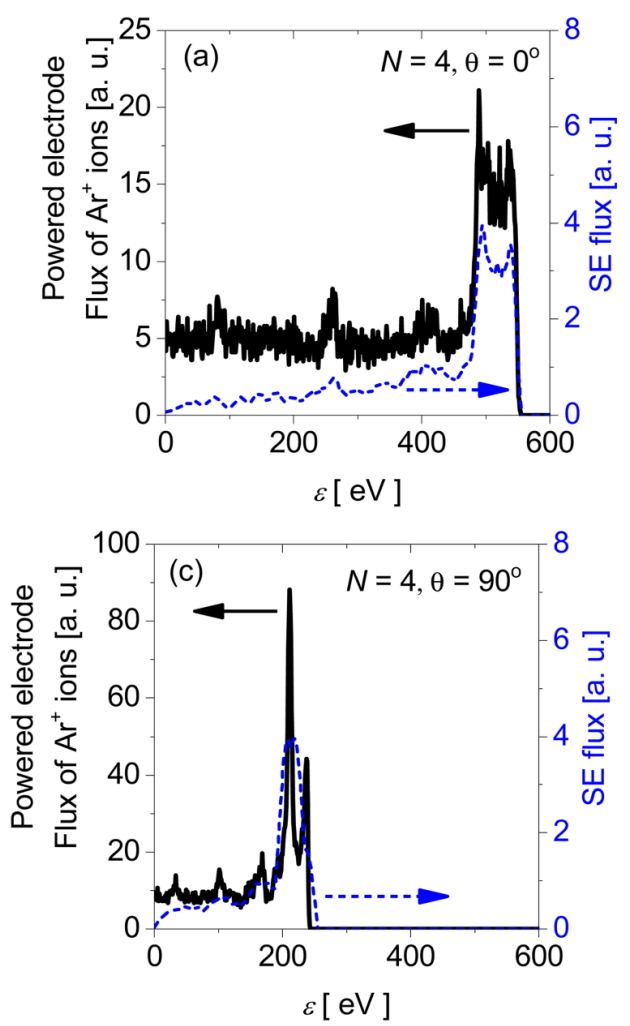
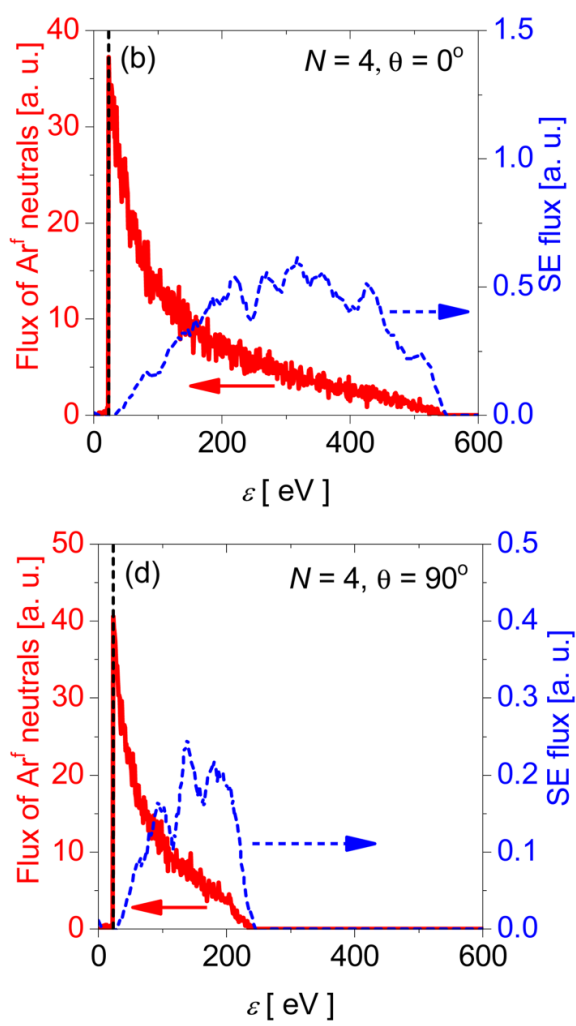

FIG. 9. Flux-energy distributions of ions (left panels) and fast atoms (right panels) at the powered electrode (solid lines, left axes) and the SE flux induced by these particles (dashed lines, right axes) as a function of the ion/atom energy for "dirty" metal surfaces, for $N=4$ harmonics, $\theta=0^{\circ}$ (top panels) and $\theta=90^{\circ}$ (bottom panels). $\gamma_{d}^{*}=0.19$ at $\theta=0^{\circ}$, and $\gamma_{d}^{*}=0.08$ at $\theta=90^{\circ}$. The dashed vertical lines in panels (b) and (d) indicate the threshold energy $(23 \mathrm{eV})$ for fast neutrals. Discharge conditions: $p=3 \mathrm{~Pa}, \phi_{\text {tot }}=800 \mathrm{~V}, \theta=\theta_{2}=\theta_{4}$, $\theta_{1}=\theta_{3}=0^{\circ}$. 


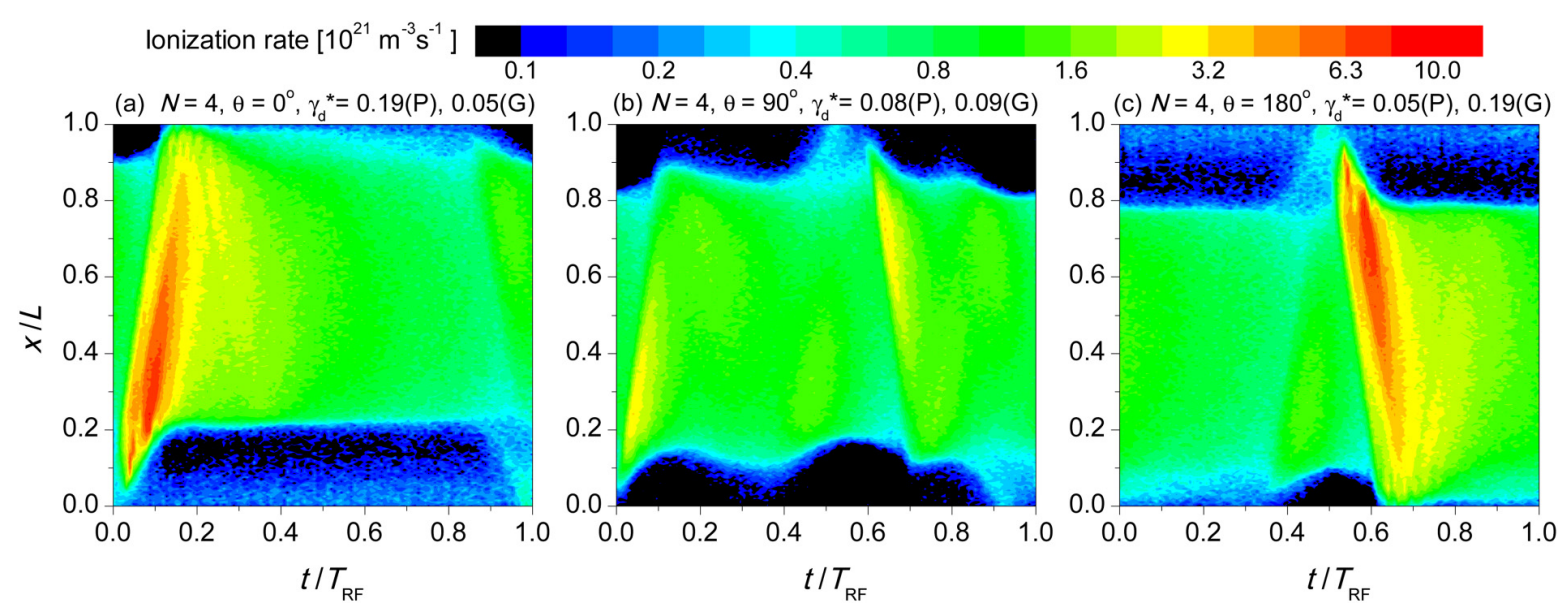

FIG. 10. Spatio-temporal plots of the ionization rate for $N=4$ harmonics, for different phase angles: $\theta=0^{\circ}$ (a), $\theta=90^{\circ}$ (b), and $\theta=180^{\circ}$ (c), obtained by assuming "dirty" surface conditions in the simulations and calculating the effective SEEC, $\gamma_{d}^{*}$, self-consistently. The values of $\gamma_{d}^{*}$ at the powered $(P)$ and grounded $(G)$ electrodes are shown in each panel's title. The powered electrode is at $x / L=0$, while the grounded electrode is at $x / L=1$. $T_{\mathrm{RF}}$ indicates one period of the fundamental frequency. Discharge conditions: $L=3 \mathrm{~cm}, p=3 \mathrm{~Pa}, \phi_{\text {tot }}=800 \mathrm{~V}, \theta=\theta_{2}=\theta_{4}, \theta_{1}=\theta_{3}=0^{\circ}$. The color scale is logarithmic.

0.05 to about 0.2 at $N=4$ ) and decreases at the powered electrode. The $\gamma_{\mathrm{d}}^{*}$ values found at the powered and grounded electrodes at $\theta=0^{\circ}$ are reversed at $\theta=180^{\circ}$.

The variation of the effective $\gamma_{\mathrm{d}}^{*}$ with $\theta$ is a result of the variation of the heavy-particle energies, and, consequently, the variation of the energy-dependent SE yields as a function of $\theta$ due to these particles (see Fig. 2). This is illustrated in Fig. 9, which shows the flux-energy distributions of ions and fast atoms at the powered electrode and the SE flux induced by these particles from "dirty" surfaces as a function of the ion/atom energy for $N=4$ harmonics, at $\theta=0^{\circ}$ and $\theta=90^{\circ}$. The relatively high value of $\gamma_{\mathrm{d}}^{*}=0.19$ at the powered electrode obtained for $N=4$ at $\theta=0^{\circ}$ (Fig. 8) is the result of the high SE yield due to ions hitting the electrode at high energies [Fig. 9(a)], aided by the contribution of energetic neutrals to the SEE [Fig. 9(b)]. At the grounded electrode, the heavy-particle energies are significantly lower [the mean ion energy is about $50 \mathrm{eV}$ at the grounded electrode compared to $325 \mathrm{eV}$ at the powered electrode, as it can be seen in Figs. 7(a) and 7(c)]. These particles induce less SEs at the grounded electrode, which explains the lower value of $\gamma_{\mathrm{d}}^{*}=$ 0.048 at the grounded electrode side.

Compared to $\theta=0^{\circ}$, the ions and fast neutrals reach the electrodes at lower energies at $\theta=90^{\circ}$ [the particle energies are below $250 \mathrm{eV}$, see the flux-energy distributions at the powered electrode in Figs. 9(c) and 9(d)]. This leads to lower SE yields based on formulas (5) and (6) for the individual heavy particles hitting the electrodes and to effective $\gamma_{\mathrm{d}}^{*}$ with values of $0.08-0.09$ at both electrodes.

For "clean" surface conditions, the self-consistently calculated effective SEEC, $\gamma_{c}^{*}$, is 0.07 (marked as a dashed line in Fig. 8) for all $N$ and $\theta$ at both electrodes. This can be explained by the energy distributions of ions and atoms bombarding the electrodes and the corresponding SE yields of these particles on "clean" surfaces. Under the conditions investigated here, the ions and atoms reach the electrodes with energies below $600 \mathrm{eV}$. At such energies, the SE yield is 0.07 for ions (Fig. 2), while even lower yields are obtained for fast neutrals based on formulas (7) and (8).

Figure 10 shows the spatio-temporal plots of the ionization rate for $N=4$, for different phase angles, $\theta=0^{\circ}, 90^{\circ}$, and $180^{\circ}$, obtained by assuming "dirty" surface conditions in the simulations and calculating the effective SEEC, $\gamma_{\mathrm{d}}^{*}$, self-consistently. At $\theta=0^{\circ}, \gamma_{\mathrm{d}}^{*}$ is high at the powered electrode $\left(\gamma_{\mathrm{d}}^{*}=0.19\right)$, while it is low at the grounded electrode $\left(\gamma_{\mathrm{d}}^{*}=0.05\right)$, see Fig. 8 . At $\theta=180^{\circ}$, the role of the electrodes is reversed, i.e., $\gamma_{\mathrm{d}}^{*}$ is low at the powered electrode while it is high at the grounded electrode side. At $\theta=90^{\circ}$, similar values of $\gamma_{\mathrm{d}}^{*}$ are obtained for both electrodes, $\gamma_{\mathrm{d}}^{*}$ is 0.08 and 0.09 at the powered and grounded electrodes, respectively. At all phase angles, the discharge operates in the $\alpha$-mode. Similar to the ionization dynamics found by assuming constant $\gamma$-coefficients in the simulations (Fig. 5), at $\theta=0^{\circ}$ and $180^{\circ}$ strong ionization at the expanding sheath edge, further enhancement by NERH is found. The $\Gamma_{\mathrm{i}}$ ion fluxes at these phase angles (Fig. 7) as well as the spatio-temporal distributions of the ionization rates [Figs. 10 (a) and $10(\mathrm{c})$ ], are similar to those obtained by assuming a constant $\gamma=0.2$ (at both electrodes) in the simulations (see for comparison Figs. 4 and 5). At $\theta=0^{\circ} / \theta=180^{\circ}$, the sheath is collapsed for a long fraction of the period of the fundamental frequency $\left(T_{\mathrm{RF}}\right)$ at the grounded/powered electrode; therefore, the SEs emitted at this electrode are accelerated by the sheath electric field only during a short fraction of $T_{\mathrm{RF}}$ and they cannot affect significantly the ionization dynamics and the plasma density. On the other hand, the sheath is expanded for a long fraction of $T_{\mathrm{RF}}$ at the opposing (powered/grounded) electrode, and the SEs emitted there contribute to the ionization; however, they also have only a limited effect on the plasma density and the ion flux at the electrodes due to the low gas pressure and their poor confinement under such conditions. 
At $\theta=90^{\circ}$, the self-consistently calculated values of $\gamma_{d}^{*}$ are much lower than 0.2 (0.08 and 0.09 at the powered and grounded electrode, respectively). The confinement of SEs is guaranteed due to the specific waveform shape at $N=4$. However, under these conditions, less SEs are emitted (indicated by the low values of $\gamma_{d}^{*}$ ) and the ionization is less efficient in the bulk region [see the ionization dynamics in Fig. 10(b) compared to Fig. 5(e)], leading to a lower plasma density and ion flux than those obtained by assuming $\gamma=0.2$ in the simulations. While in the latter case a nearly constant flux of ions at the electrodes is found as a function of $\theta$ (and the mean ion energy can be tuned by $\theta$, i.e., the separate control of ion properties is possible), this is not the case when the SEE is described realistically in the simulations and the SEEC for dirty surface conditions, $\gamma_{\mathrm{d}}^{*}$, is calculated self-consistently in the simulations. (The same can be stated for clean surfaces too.)

The results presented in this section confirmed that the surface conditions affect significantly the quality of the separate control of ion properties at the electrodes in low-pressure discharges driven by TVWs. Important differences from the results obtained by assuming constant values for the ion-induced SEEC, $\gamma$, are found by taking into account the surface conditions and the energy dependence of the SE yield due to heavy-particle impact in the simulations. For specific values of $\gamma$, the simulations predict a good quality of the separate control of ion properties at the electrodes by tuning the control parameter for the mean ion energy, $\theta$, while the simulations which operate with energy-dependent SE yields for heavy particles show that the separate control of the ion properties is not possible under the conditions studied here.

\section{B. High-pressure regime (100 Pa)}

In this section, the results obtained for $100 \mathrm{~Pa}$ and $\phi_{\text {tot }}=120 \mathrm{~V}$ are presented. Similar to Sec. III A on low pressures $(3 \mathrm{~Pa})$, results obtained by using constant values for the ion-induced SEEC, $\gamma$, are presented first, followed by results based on simulations with a selfconsistent calculation of the effective SEECs, $\gamma_{d}^{*}$ and $\gamma_{c}^{*}$, for "dirty" and "clean" surfaces.

\section{Constant heavy-particle induced SEECs, $\gamma$, at $100 \mathrm{~Pa}$}

Figure 11 shows the dc self-bias voltage, $\eta$, as a function of $\theta$, for $N=1,2$, and 4 harmonics, assuming $\gamma=0.0,0.2$, and 0.4 as ion-induced SE yield. $\eta$ can be tuned efficiently by changing $\theta$, and increasing $N$ enlarges its control interval. This is similar to the scenario seen at the low pressure of $3 \mathrm{~Pa}$ (Fig. 3). However, at $3 \mathrm{~Pa}$ almost the same values of $\eta$ were obtained for the different values of $\gamma$ at a given $\theta$ (max. 5\% difference). Here, the choice of $\gamma$ affects significantly the $\mathrm{dc}$ self-bias voltage. At $N=2$, for $\gamma=0.0,|\eta|$ reaches a maximum value of about $30 \mathrm{~V}$, while at $\gamma=0.4$ the maximum of $|\eta|$ is about $20 \mathrm{~V}$ (a decrease of $|\eta|$ by about 33\%). (This corresponds to a decrease of the relative amplitude of the dc self-bias, $\eta / \phi_{\text {tot }}$ from $25 \%$ to about $17 \%$.) At $N=4$, the dc self-bias voltage can be tuned from about $-40 \mathrm{~V}$ to $40 \mathrm{~V}$ at $\gamma=0.0$ and from $-30 \mathrm{~V}$ to $30 \mathrm{~V}$ at $\gamma=0.4$ by changing $\theta$ from $0^{\circ}$ to $180^{\circ}$, i.e., $|\eta|$ decreases by about $25 \%\left(\eta / \phi_{\text {tot }}\right.$ decreases from $33 \%$ to $\left.25 \%\right)$. These trends can be explained based on the ionization dynamics and operation of the discharge in the hybrid $\alpha-\gamma$ mode or

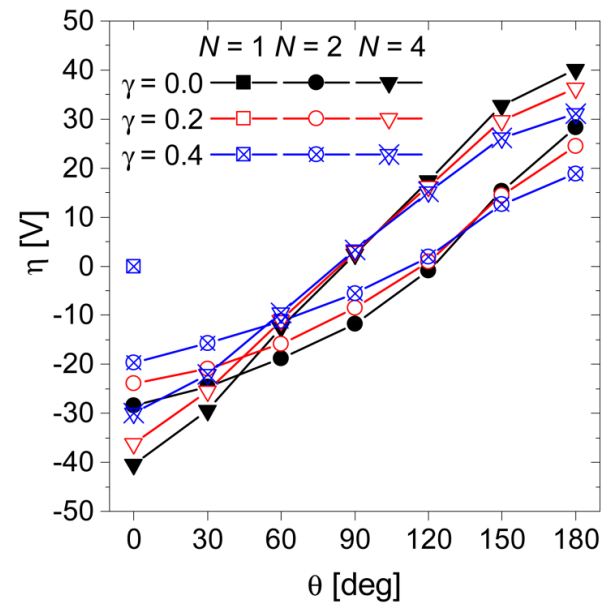

FIG. 11. Dependence of the dc self-bias voltage, $\eta$, on the identical phase angle of the even harmonics, $\theta=\theta_{2}=\theta_{4}\left(\theta_{1}=\theta_{3}=0^{\circ}\right)$, for different numbers of applied harmonics, $N$, and different values of the $\gamma$-coefficient $(\gamma=0.0$, $0.2,0.4)$. Discharge conditions: $p=100 \mathrm{~Pa}, \phi_{\text {tot }}=120 \mathrm{~V}$. Note that the symbols completely overlap in some cases, e.g., at $N=1$.

$\gamma$-mode at high values of $\gamma$ instead of the pure $\alpha$-mode at $\gamma=0.0$, see later in this section.

Figure 12 shows the mean ion energy [panels (a) and (c)] and the ion fluxes [panels (b) and (d)] at the grounded and powered electrodes for different numbers of applied harmonics and different values of the $\gamma$-coefficient. For a given $N \geq 2$ and $\gamma,\left\langle E_{\mathrm{i}}\right\rangle$ can be controlled efficiently by tuning $\theta$. At $N=2,\left\langle E_{\mathrm{i}}\right\rangle$ can be changed by a factor of about 1.6 at both electrodes by changing $\theta$, while by adding more harmonics to the driving voltage waveform the control range is slightly enhanced, and at $N=4$ a change of $\left\langle E_{\mathrm{i}}\right\rangle$ by a factor of maximum 2.3 can be achieved [Figs. 12(a) and 12(c)]. Independently of the choice of $\gamma$, for a given $N \geq 2$, the ion flux does not remain constant as a function of $\theta$ under these conditions: $\Gamma_{\mathrm{i}}$ decreases at the powered electrode [Fig. 12(d)], while it increases at the grounded electrode [Fig. 12(b)]. For a given $\theta$ and $N$, higher ion fluxes are obtained for higher values of $\gamma$. However, the choice of $\gamma$ does not change the quality of the separate control of ion properties, $\Gamma_{\mathrm{i}}$ and $\left\langle E_{\mathrm{i}}\right\rangle$ are coupled at such high pressures for all $\gamma$. This is different compared to the results obtained for $3 \mathrm{~Pa}$ (Fig. 4), where for specific choices of $\gamma,\left\langle E_{\mathrm{i}}\right\rangle$ can be tuned by $\theta$ independently of $\Gamma_{\mathrm{i}}$, and changes of the $\gamma$-coefficient affect this control significantly. A detailed analysis of the ionization dynamics for the same discharge conditions, for $\gamma=0.4$. was performed previously in Ref. 51-that study has pointed out that these differences are caused by the different electron power absorption and ionization modes present at $3 \mathrm{~Pa}$ and at $100 \mathrm{~Pa}$. In the $\alpha$-mode operation at $3 \mathrm{~Pa}$, the ionization dynamics is nonlocal (Fig. 5), while at $100 \mathrm{~Pa}$, where $\gamma$-mode operation develops, the ionization is localized at the sheath edges (see Fig. 13) and both the ion flux and the mean ion energy depend on the sheath voltage. Due to the waveform shape at a high number of applied harmonics (Fig. 1), the sheath voltage is maximum at the powered electrode side for a long fraction of $T_{\mathrm{RF}}$. This results in 

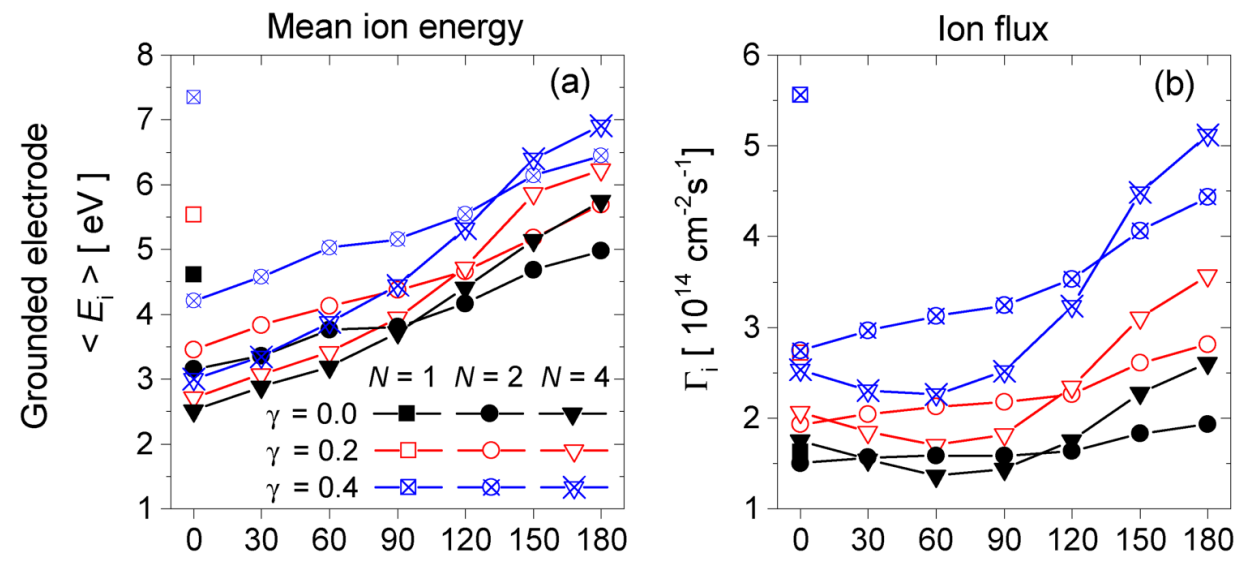

FIG. 12. Mean ion energy (panels on the left), $\left\langle E_{\mathrm{i}}\right\rangle$, and ion flux (panels on the right), $\Gamma_{\mathrm{i}}$, at the grounded and powered electrodes (top and bottom panels, respectively) as a function of $\theta=\theta_{2}=\theta_{4} \quad\left(\theta_{1}=\theta_{3}=0^{\circ}\right) \quad$ for
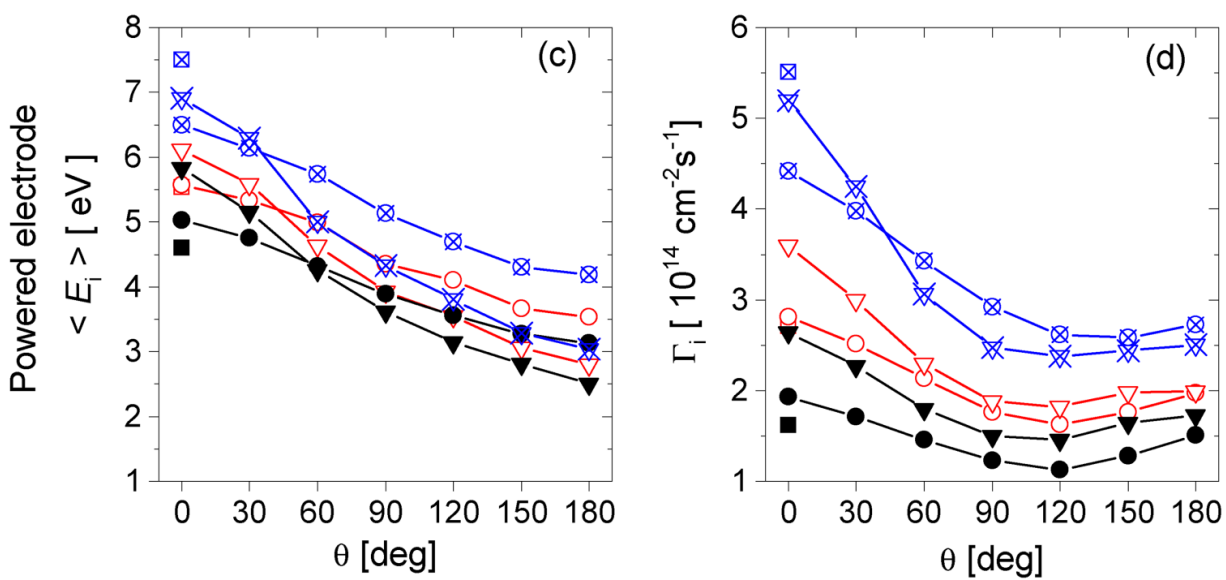
different numbers of applied harmonics, $N$, and different values of the $\gamma$-coefficient $(\gamma=0.0,0.2,0.4)$. Discharge conditions: $p=100 \mathrm{~Pa}$, $\phi_{\text {tot }}=120 \mathrm{~V}$. Note that the symbols completely overlap in some cases.

ionization induced by SEs at the powered electrode during a longer fraction of $T_{\mathrm{RF}}$ compared to the grounded electrode side, which finally leads to an asymmetric ionization profile and a higher plasma density at the powered electrode compared to the grounded one. This is the reason why $\Gamma_{\mathrm{i}}$ is higher at the powered electrode compared to the grounded electrode at $\theta=0^{\circ}$ and higher at the grounded electrode at $\theta=180^{\circ}$ compared to the powered electrode [Figs. 12(b) and 12(d)]. As tuning $\theta$ changes the symmetry of the spatio-temporal distribution of the ionization, mainly concentrated in the sheath regions (see Fig. 13 for $N=4$ ), the ion flux does not remain constant as a function of $\theta$ at both electrodes. This effect is most pronounced at high numbers of applied harmonics and high values of the $\gamma$-coefficient. For $N=2, \Gamma_{\mathrm{i}}$ changes by a factor of about 1.3 and 1.6 at the electrodes by changing $\theta$ from $0^{\circ}$ to $180^{\circ}$, for $\gamma=0.0$ and $\gamma=0.4$, respectively. For $N=4, \Gamma_{\mathrm{i}}$ changes by a factor of about 1.5 and 2 at the electrodes by changing $\theta$ from $0^{\circ}$ to $180^{\circ}$, for $\gamma=0.0$ and $\gamma=0.4$, respectively.

\section{Realistic heavy-particle induced SEECs, $\gamma^{*}$, at $100 \mathrm{~Pa}$}

The results of the simulations in which the SE yields were calculated as a function of the heavy-particle energies at $3 \mathrm{~cm}, 100 \mathrm{~Pa}$, and $\phi_{\text {tot }}=120 \mathrm{~V}$, are presented below. Similar to the study for the low-pressure regime, both "dirty" and "clean" surface conditions are covered here, and the $\gamma_{\mathrm{d}}^{*}$ and $\gamma_{\mathrm{c}}^{*}$ effective SEECs are calculated self-consistently for these surface conditions.

Figure 14 shows the dc self-bias voltage, $\eta$, as a function of the identical phase angle of the even harmonics, $\theta=\theta_{2}=\theta_{4}$, for different numbers of applied harmonics, $N$, for "dirty" and "clean" surface conditions. The dc self-bias voltages obtained for "dirty" surfaces agree with those obtained for "clean" surfaces, as it was found also at the low pressure of $3 \mathrm{~Pa}$ (Fig. 6). Similar to the results shown in Fig. 11 for constant $\gamma$ ion-induced SEECs, the dc self-bias voltage can be tuned efficiently by changing $\theta$, and the $|\eta|$ values at both $N=2$ and $N=4$ are close to those obtained by assuming a constant $\gamma$-coefficient of 0.0 in the simulations (this can be explained by the relatively low values of $\gamma_{d}^{*}$ and $\gamma_{c}^{*}$ under these conditions, see later in Fig. 16). At $N=2, \eta$ can be tuned within about $\pm 28 \mathrm{~V}$, while at $N=4$ the dc self-bias voltage can be changed within about $\pm 40 \mathrm{~V}$ by tuning $\theta$ from $0^{\circ}$ to $180^{\circ}$.

Figure 15 shows the mean ion energy, $\left\langle E_{\mathrm{i}}\right\rangle$, and the ion flux, $\Gamma_{\mathrm{i}}$, at the grounded and powered electrodes as a function of $\theta$ for different $N$, for "dirty" (filled symbols) and "clean" (open symbols) surface conditions. For both conditions, $\left\langle E_{\mathrm{i}}\right\rangle$ can be increased/ decreased by a factor of about 1.6 at $N=2$ and by a factor of about 

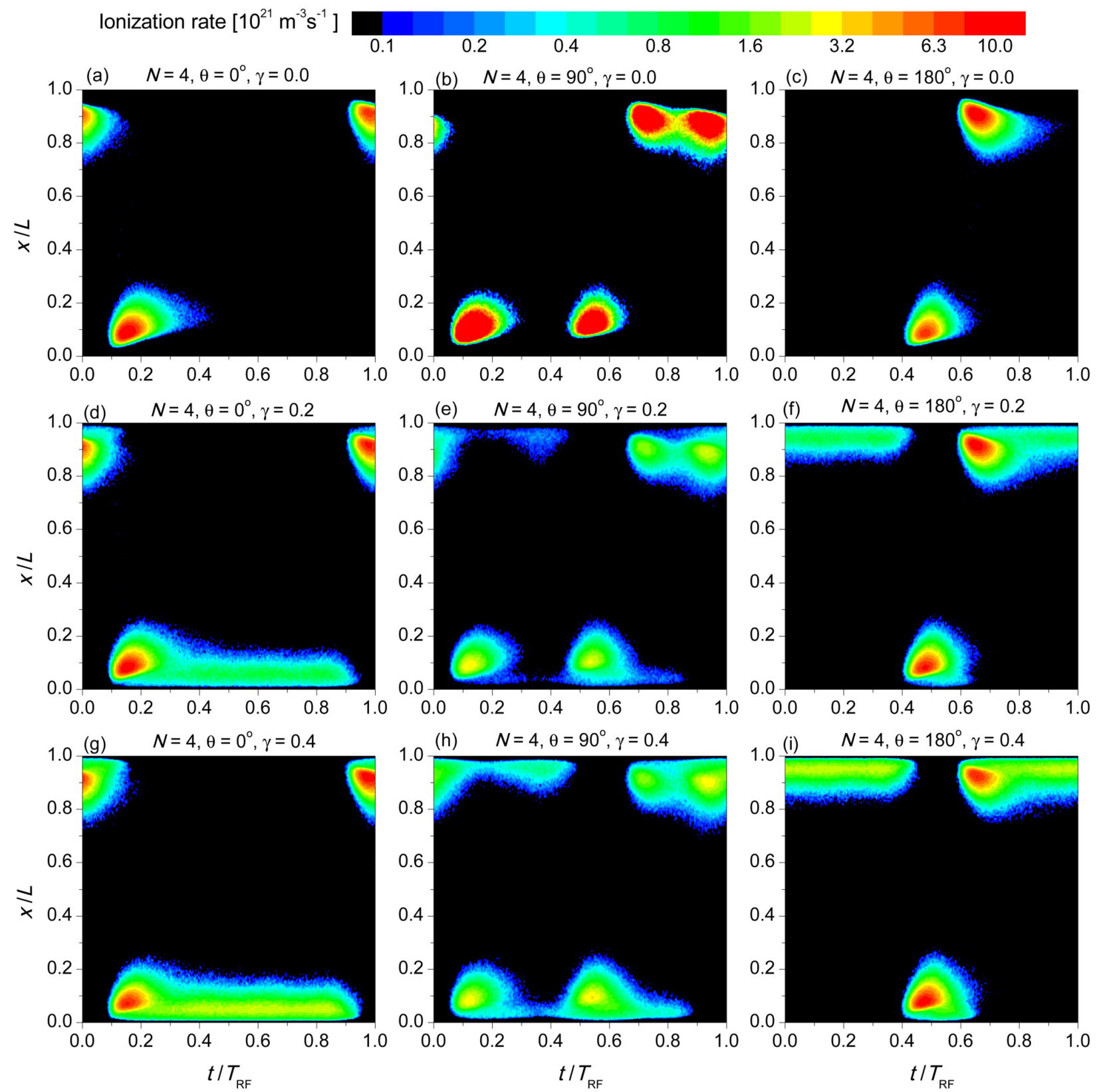

FIG. 13. Spatio-temporal plots of the ionization rate for $N=4$ harmonics, for different phase angles: $\theta=0^{\circ}$ (left column), $\theta=90^{\circ}$ (center column), and $\theta=180^{\circ}$ (right column) and for different values of the $\gamma$-coefficient: $\gamma=0.0$ (top row), $\gamma=0.2$ (center row), and $\gamma=0.4$ (bottom row). The powered electrode is at $x / L=0$, while the grounded electrode is at $x / L=1$. $T_{\mathrm{RF}}$ indicates one period of the fundamental frequency. Discharge conditions: $L=3 \mathrm{~cm}, p=100 \mathrm{~Pa}, \phi_{\text {tot }}=120 \mathrm{~V}, \theta=\theta_{2}=\theta_{4}$, $\theta_{1}=\theta_{3}=0^{\circ}$ ). The color scale is logarithmic.

2.3 at $N=4$ at the grounded/powered electrode by changing $\theta$ from $0^{\circ}$ to $180^{\circ}$ [these results agree with those obtained for $\gamma=0.0$, see Figs. 15(a) and 15(c) compared to Figs. 12(a) and 12(c)]. The $\left\langle E_{\mathrm{i}}\right\rangle$ energy values for "clean" surfaces are slightly higher (a maximum difference of $5 \%$ can be seen). The ion flux changes by tuning $\theta$ at both electrodes, coupled to the mean ion energy, for both "dirty" and "clean" surfaces [Figs. 15(b) and 15(d)]. At $N=2, \Gamma_{\mathrm{i}}$ changes by about $30 \%$, while it changes by about $60 \%$ at $N=4$ by tuning 


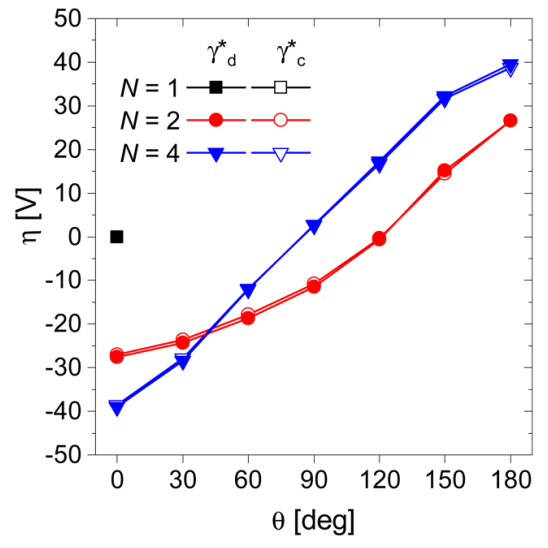

FIG. 14. Dependence of the dc self-bias voltage, $\eta$, on the identical phase angle of the even harmonics, $\theta=\theta_{2}=\theta_{4} \quad\left(\theta_{1}=\theta_{3}=0^{\circ}\right)$, for different numbers of applied harmonics, $N$, obtained from simulations with selfconsistently calculated effective SEECs, $\gamma_{d}^{*}$ and $\gamma_{c}^{*}$, for "dirty" (filled symbols) and "clean" (open symbols) surface conditions, respectively. Discharge conditions: $p=100 \mathrm{~Pa}, \phi_{\text {tot }}=120 \mathrm{~V}$. Note that the symbols completely overlap in some cases, e.g., at $N=1$.

$\theta$ from $0^{\circ}$ to $180^{\circ}$, for both "dirty" and "clean" surfaces (these results also agree with those obtained for $\gamma=0.0$ ). For "clean" surfaces, higher (up to 12\%) ion fluxes are obtained for a given $\theta$ and $N$ compared to "dirty" surfaces (this is different from the scenario at low pressure, where higher ion fluxes were obtained for "dirty" surfaces compared to "clean" surfaces). This can be explained based on the self-consistently calculated values of the effective SEECs, $\gamma_{\mathrm{d}}^{*}$ and $\gamma_{\mathrm{c}}^{*}$.

In the case of "clean" surfaces, $\gamma_{c}^{*}=0.07$ for all $N$ and $\theta$, while in the case of "dirty" surfaces even smaller effective SEECs are obtained. $\gamma_{\mathrm{d}}^{*}$ changes as a function of $\theta$ at both electrodes (Fig. 16). By tuning $\theta$ from $0^{\circ}$ to $180^{\circ}, \gamma_{\mathrm{d}}^{*}$ increases at the grounded electrode (from about 0.013 to about 0.17 at $N=2$ and from about 0.011 to about 0.018 at $N=4$ ) and decreases at the powered electrode. The $\gamma_{d}^{*}$ values found at the powered and grounded electrodes at $\theta=0^{\circ}$ are reversed at $\theta=180^{\circ}$. As explained above for the low-pressure case, the dependence of $\gamma_{\mathrm{d}}^{*}$ on $\theta$ arises from the dependence of the SE yields on the energies of the heavy particles bombarding the electrodes (see Fig. 2). However, the particle energies are low under high-pressure conditions at $\phi_{\text {tot }}=120 \mathrm{~V}$ [the maximum of the mean ion energy is about $6 \mathrm{eV}$ at both electrodes, see Figs. 15(a) and 15(c)], resulting in small values of the effective SEECs for both "dirty" and "clean" surface conditions.

The simulations performed at $100 \mathrm{~Pa}$ with constant ioninduced SEECs, as well as with realistic self-consistently calculated SEECs, result in the same trends of the ion properties at the electrodes as a function of the control parameter for the mean ion energy, $\theta$. All simulations show that the mean ion energy and the ion flux are coupled as $\theta$ changes, i.e., the separate control of the ion properties cannot be achieved under the conditions studied here. For "dirty" surfaces, $\gamma_{d}^{*}$ changes as a function of $\theta$ at both electrodes, while in the case of "clean"
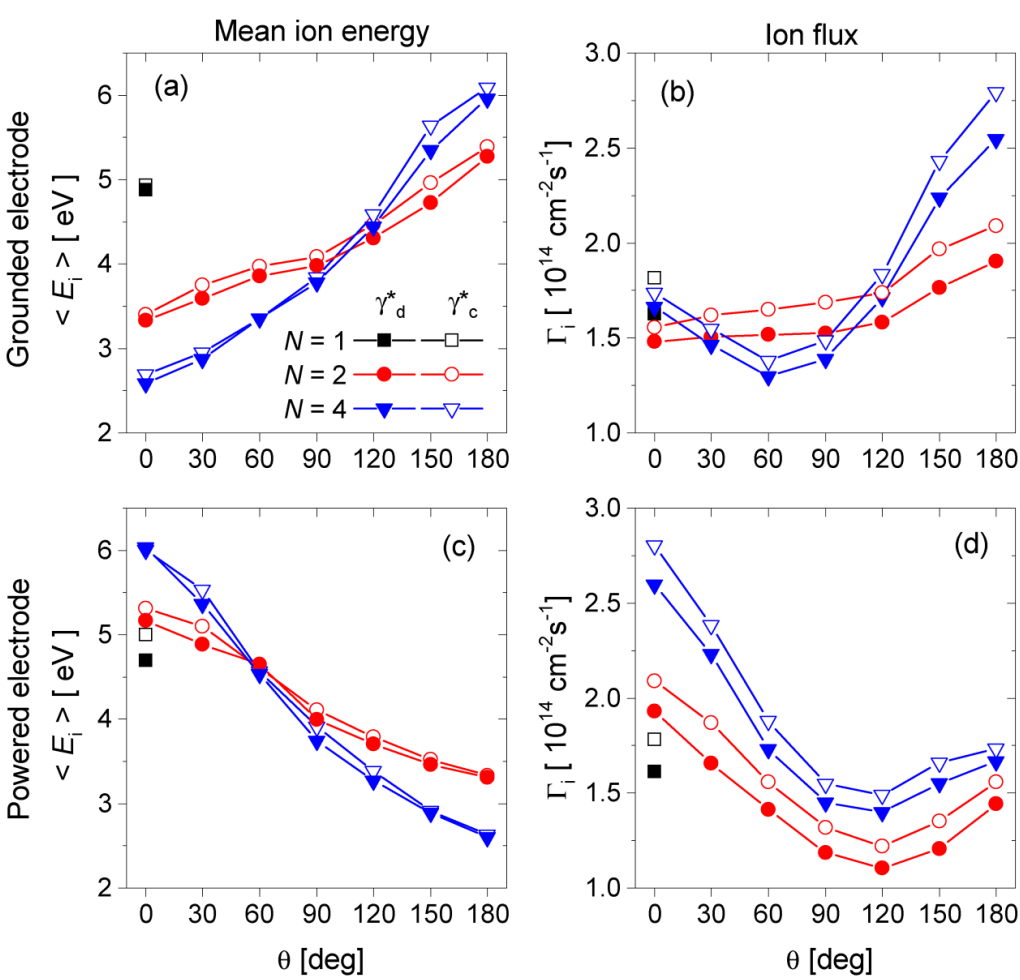

FIG. 15. Mean ion energy (panels on the left), $\left\langle E_{\mathrm{i}}\right\rangle$, and ion flux (panels on the right), $\Gamma_{\mathrm{i}}$, at the grounded and powered electrodes (top and bottom panels, respectively) as a function of $\theta=\theta_{2}=\theta_{4}\left(\theta_{1}=\theta_{3}=0^{\circ}\right)$ for different numbers of applied harmonics, $N$, obtained from simulations with self-consistently calculated effective SEECs, $\gamma_{d}^{*}$ and $\gamma_{c}^{*}$, for "dirty" (filled symbols) and "clean" (open symbols) surface conditions, respectively. Discharge conditions: $p=100 \mathrm{~Pa}, \phi_{\text {tot }}=120 \mathrm{~V}$. Note that the symbols completely overlap in some cases. 


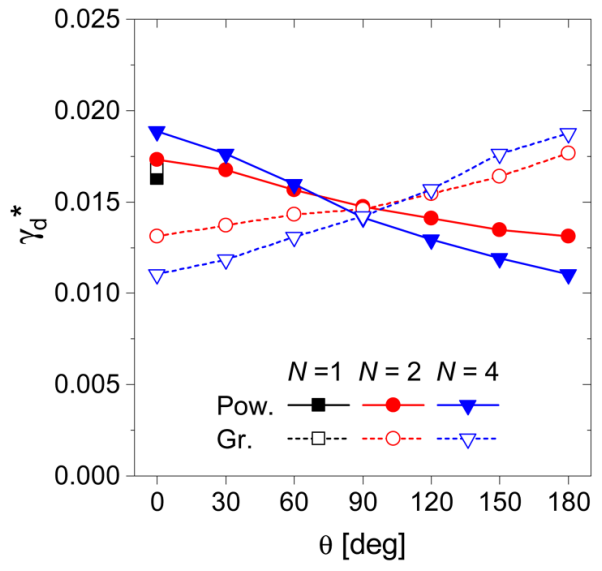

FIG. 16. Self-consistently calculated effective SEEC for "dirty" surface conditions, $\gamma_{d}^{*}$, at the powered and grounded electrodes (filled and open symbols, respectively) as a function of $\theta=\theta_{2}=\theta_{4} \quad\left(\theta_{1}=\theta_{3}=0^{\circ}\right)$ for different numbers of applied harmonics, $N$. The effective SEEC for "clean" surface conditions (not shown in the figure), $\gamma_{c}^{*}$, is 0.07 for all $\theta$ and $N$ at both electrodes. Discharge conditions: $p=100 \mathrm{~Pa}, \phi_{\text {tot }}=120 \mathrm{~V}$.

surfaces $\gamma_{c}^{*}$ is 0.07 for all $\theta$ and $N$. However, for both surface conditions, the self-consistently calculated SEECs at $100 \mathrm{~Pa}$ are small ( 0.07 for "clean" surfaces and below 0.02 for dirty surfaces), i.e., the values obtained for $\gamma_{d}^{*}$ and $\gamma_{c}^{*}$ are significantly lower than those typically assumed in PIC/MCC simulations of CCPs in Ar under similar discharge conditions.

\section{CONCLUSIONS}

Particle-in-Cell/Monte Carlo Collision simulations were performed to investigate the effects of heavy-particle induced SEs on the ionization dynamics and on the control of ion properties at the electrodes in geometrically symmetric capacitively coupled discharges in argon driven by tailored voltage waveforms. The driving voltage waveform, composed of a maximum of four consecutive harmonics $(1 \leq N \leq 4)$ of the fundamental frequency, $f=13.56 \mathrm{MHz}$, was tailored by adjusting the phases of the even harmonics $\left(\theta_{2}=\theta_{4}=\theta\right.$ varied from $0^{\circ}$ to $\left.180^{\circ}\right)$, while the phases of the odd harmonics were fixed $\left(\theta_{1}=\theta_{3}=0^{\circ}\right)$. The simulations were carried out at neutral gas pressures of $3 \mathrm{~Pa}$ (nearly collisionless low-pressure regime) with $\phi_{\text {tot }}=800 \mathrm{~V}$ and at $100 \mathrm{~Pa}$ (collisional high-pressure regime) with $\phi_{\text {tot }}=120 \mathrm{~V}$. At both pressures, different approaches have been adopted in the simulations to describe the SEE induced by heavy particles at the electrodes: (i) constant ion-induced SEECs, $\gamma$, were used (assuming values for $\gamma$ between 0.0 and 0.4 ) and (ii) realistic, energy-dependent SE yields for ions and fast neutrals.

At both pressures, a dc self-bias, $\eta$, was found to be generated for $N \geq 2$ via the EAE. $\eta$ can be tuned efficiently by $\theta$, and adding more harmonics to the driving voltage waveform enlarges the control interval of $\eta$. The simulations showed that the mean ion energy at the electrodes, $\left\langle E_{\mathrm{i}}\right\rangle$, can be controlled by $\theta$, under all conditions investigated here.
At the low pressure of $3 \mathrm{~Pa}$, depending on the value of the $\gamma$-coefficient, largely different dependencies of the ion flux at the electrodes, $\Gamma_{\mathrm{i}}$, on the control parameter for the mean ion energy, $\theta$, were obtained. For $\gamma=0.2, \Gamma_{\mathrm{i}}$ remained essentially constant as a function of $\theta$, independently of the choice of $N$, i.e., the mean ion energy could be controlled separately from the ion flux by adjusting $\theta$. This had been previously demonstrated in Ref. 51 . However, the present simulations showed that for values of $\gamma$ different from 0.2 the quality of the separate control of the ion properties changes significantly. Using lower/higher values for $\gamma(0.0$ and 0.3 , respectively) in the simulations at $3 \mathrm{~Pa}$ resulted in varying ion fluxes as a function of $\theta$, i.e., both $\Gamma_{\mathrm{i}}$ and $\left\langle E_{\mathrm{i}}\right\rangle$ changed by tuning $\theta$. Under such conditions, the separate control of $\left\langle E_{\mathrm{i}}\right\rangle$ and $\Gamma_{\mathrm{i}}$ could not be realized.

At the high pressure of $100 \mathrm{~Pa}$, independently of the choice of $\gamma$, for a given $N \geq 2$, the ion flux did not remain constant as a function of $\theta: \Gamma_{\mathrm{i}}$ decreased at the powered electrode, while it increased at the grounded electrode. For a given $\theta$ and $N$, higher ion fluxes were obtained for high values of $\gamma$. However, the choice of $\gamma$ did not change the quality of the separate control of ion properties, $\Gamma_{\mathrm{i}}$ and $\left\langle E_{\mathrm{i}}\right\rangle$ were coupled at such high pressures for all $\gamma$. This was different compared to the results obtained for $3 \mathrm{~Pa}$, where for specific choices of $\gamma,\left\langle E_{\mathrm{i}}\right\rangle$ could be tuned by $\theta$ independently of $\Gamma_{\mathrm{i}}$, and changes of the $\gamma$-coefficient affected this control significantly.

At both pressures, the surface conditions affected the plasma parameters and the quality of the separate control of ion properties at the electrodes. Important differences from the results obtained by assuming constant values for the ion-induced SEEC, $\gamma$, were found by taking into account the surface conditions and the energy dependence of the SE yield due to heavy-particle impact in the simulations. For "clean" surface conditions, the self-consistently calculated $\gamma_{c}^{*}$ was found to be 0.07 for all conditions studied here. For "dirty" surface conditions, $\gamma_{\mathrm{d}}^{*}$ changed as a function of $\theta$ at both electrodes: by tuning $\theta$ from $0^{\circ}$ to $180^{\circ}, \gamma_{\mathrm{d}}^{*}$ increased at the grounded electrode and decreased at the powered electrode.

\section{ACKNOWLEDGMENTS}

This work was supported by the U.S. NSF under Grant No. PHY. 1601080, by the German Research Foundation (DFG) within the frame of the collaborative research center SFB-TR 87 (project C1), by the Hungarian National Research, Development and Innovation Office via Grant Nos. K-119357, PD-121033, and FK-128924, and by the J. Bolyai Research Fellowship of the Hungarian Academy of Sciences.

\section{REFERENCES}

${ }^{1}$ M. A. Lieberman and A. J. Lichtenberg, Principles of Plasma Discharges and Materials Processing, 2nd ed. (Wiley, New York, 2005).

${ }^{2}$ T. Makabe and Z. Petrović, Plasma Electronics: Applications in Microelectronic Device Fabrication (Taylor \& Francis, London, 2006).

${ }^{3}$ P. Chabert and N. Braithwaite, Physics of Radio-Frequency Plasmas (Cambridge University Press, Cambridge, 2011).

${ }^{4}$ B. G. Heil, U. Czarnetzki, R. P. Brinkmann, and T. Mussenbrock, J. Phys. D Appl. Phys. 41, 165202 (2008).

5. Schulze, E. Schüngel, Z. Donkó, and U. Czarnetzki, Plasma Sources Sci. Technol. 20, 015017 (2011).

${ }^{6}$ T. Lafleur, R. W. Boswell, and J. P. Booth, Appl. Phys. Lett. 100, 194101 (2012).

${ }^{7}$ E. V. Johnson, P. A. Delattre, and J. P. Booth, Appl. Phys. Lett. 100, 133504 (2012). 
${ }^{8}$ S. Bienholz, T. Styrnoll, and P. Awakowicz, J. Phys. D Appl. Phys. 47, 065201 (2014). ${ }^{9}$ P. Diomede, D. J. Economou, T. Lafleur, J. P. Booth, and S. Longo, Plasma Sources Sci. Technol. 23, 065049 (2014).

${ }^{10}$ B. Bruneau, T. Gans, D. O'Connell, A. Greb, E. V. Johnson, and J. P. Booth, Phys. Rev. Lett. 114, 125002 (2015).

${ }^{11}$ T. Lafleur, Plasma Sources Sci. Technol. 25, 013001 (2016).

${ }^{12}$ C. K. Birdsall and A. B. Langdon, Plasma Physics via Computer Simulation (McGraw-Hill, New York, 1985).

${ }^{13}$ R. W. Hockney and J. W. Eastwood, Computer Simulation Using Particles (McGraw-Hill, New York, 1981).

${ }^{14}$ C. K. Birdsall, IEEE Trans. Plasma Sci. 19, 65 (1991).

${ }^{15}$ P. Diomede, M. Capitelli, and S. Longo, Plasma Sources Sci. Technol. 14, 459 (2005).

${ }^{16}$ K. Matyash, R. Schneider, F. Taccogna, A. Hatazarna, S. Longo, M. Capitelli, D. Tskhakaya, and F. X. Bronold, Contrib. Plasma Phys. 47, 595 (2007).

17 J. P. Verboncoeur, Plasma Phys. Control Fusion 47, A231 (2005).

18Z. Donkó, Plasma Sources Sci. Technol. 20, 024001 (2011).

${ }^{19}$ Z. Donkó, J. Schulze, P. Hartmann, I. Korolov, and E. Schüngel, Plasma Phys. Control Fusion 54, 124003 (2012).

${ }^{\mathbf{2 0}}$ A. Derzsi, I. Korolov, E. Schüngel, Z. Donkó, and J. Schulze, Plasma Sources Sci. Technol. 24, 034002 (2015).

${ }^{21}$ I. Korolov, A. Derzsi, Z. Donkó, E. Schüngel, and J. Schulze, Plasma Sources Sci. Technol. 25, 015024 (2016).

${ }^{22}$ O. Braginsky, A. Kovalev, D. Lopaev, O. Proshina, T. Rakhimova, A. Vasilieva, D. Voloshin, and S. Zyryanov, J. Phys. D 45, 015201 (2012).

${ }^{23}$ A. Bojarov, M. Radmilović-Radjenović, and Z. Lj. Petrović, in Proceedings of 20th ESCAMPIG, Novi Sad, Serbia, 13-17 July 2010 (2010), P2.38; Publications of the Astronomical Observatory of Belgrade, No. 89131 (2010); in Proceedings of 65th Annual Gaseous Electronics Conference, Austin, TX, 22-26 October 2012 (2012); in Proceedings of 27th Summer School and International Symposium on the Physics of Ionized Gases, Belgrade, Serbia, 26-29 August 2014 (2014).

${ }^{24}$ M. Radmilović-Radjenović and Z. Lj. Petrović, Eur. Phys. J. D 54, 445 (2009).

${ }^{25} \mathrm{~J}$. T. Gudmundsson, E. Kawamura, and M. A. Lieberman, Plasma Sources Sci. Technol. 22, 035011 (2013).

${ }^{\mathbf{2 6}}$ H. Hannesdottir and J. T. Gudmundsson, Plasma Sources Sci. Technol. 25, 055002 (2016).

${ }^{27}$ A. Greb, K. Niemi, D. O'Connell, and T. Gans, Appl. Phys. Lett. 103, 244101 (2013).

${ }^{28}$ P. Belenguer and J. P. Boeuf, Phys. Rev. A 41, 4447 (1990).

${ }^{29}$ J. Schulze, B. G. Heil, D. Luggenhölscher, and U. Czarnetzki, IEEE Trans. Plasma Sci. 36, 1400 (2008).

${ }^{30}$ J. Schulze, B. G. Heil, D. Luggenhölscher, R. P. Brinkmann, and U. Czarnetzki, J. Phys. D 41, 195212 (2008).

${ }^{31}$ J. Schulze, T. Kampschulte, D. Luggenhölscher, R. P. Brinkmann, and U. Czarnetzki, J. Phys. Conf. Ser. 86, 12010 (2008).

${ }^{32}$ M. M. Turner, J. Phys. D 42, 194008 (2009).

${ }^{33}$ T. Mussenbrock, R. P. Brinkmann, M. A. Lieberman, A. J. Lichtenberg, and E. Kawamura, Phys. Rev. Lett. 101, 085004 (2008).

${ }^{34}$ V. A. Schweigert, A. L. Alexandrov, S. F. Gimelshtein, and M. S. Ivanov, Plasma Sources Sci. Technol. 8, B1 (1999).

${ }^{35}$ Z. Donkó, J. Schulze, P. Hartmann, I. Korolov, U. Czarnetzki, and E. Schüngel, Appl. Phys. Lett. 97, 081501 (2010).
${ }^{36}$ J. Schulze, Z. Donkó, E. Schüngel, and U. Czarnetzki, Plasma Sources Sci. Technol. 20, 045007 (2011).

${ }^{37}$ M. M. Turner, P. Chabert, P. Levif, P. Boyle, and J. Robiche, in Proceedings of 18th ICPIG (Prague, Czech Republic, 2007), G08.

${ }^{38}$ J. P. Booth, G. Curley, D. Marić, and P. Chabert, Plasma Sources Sci. Technol. 19, 015005 (2010).

${ }^{39}$ C. Böhm and J. Perrin, Rev. Sci. Instrum. 64, 31 (1993).

${ }^{40}$ Q. Liu, Y. Liu, T. Samir, and Z. Ma, Phys. Plasmas 21, 083511 (2014).

${ }^{41}$ T. Lafleur, P. Chabert, and J. P. Booth, J. Phys. D Appl. Phys. 46, 135201 (2013).

${ }^{42}$ I. Korolov, A. Derzsi, Z. Donkó, and J. Schulze, Appl. Phys. Lett. 103, 064102 (2013).

${ }^{43}$ U. Czarnetzki, B. G. Heil, J. Schulze, Z. Donkó, T. Mussenbrock, and R. P. Brinkmann, J. Phys. Conf. Ser. 162, 012010 (2009).

${ }^{44}$ M. Daksha, A. Derzsi, S. Wilczek, J. Trieschmann, T. Mussenbrock, P. Awakowicz, Z. Donkó, and J. Schulze, Plasma Sources Sci. Technol. 26, 085006 (2017).

${ }^{45}$ J. T. Gudmundsson and A. Proto, Plasma Sources Sci. Technol. 28, 045012 (2019).

${ }^{46}$ A. V. Phelps and Z. Lj. Petrović, Plasma Sources Sci. Technol. 8, R21 (1999).

${ }^{47}$ Z. Donkó, J. Appl. Phys 88, 2226 (2000).

${ }^{48}$ Z. Donkó, Phys. Rev. E 64, 026401 (2001).

${ }^{49}$ M. Daksha, B. Berger, E. Schüngel, I. Korolov, A. Derzsi, M. Koepke, Z. Donkó, and J. Schulze, J. Phys. D Appl. Phys. 49, 234001 (2016).

${ }^{50}$ M. Daksha, A. Derzsi, Z. Donkó, and J. Schulze, Plasma Sources Sci. Technol. 28, 034002 (2019).

${ }^{51}$ A. V. Derzsi, I. Korolov, E. Schuengel, Z. Donkó, and J. Schulze, Plasma Sources Sci. Technol. 22, 065009 (2013).

${ }^{52}$ A. V. Phelps, see http://jilawww.colorado.edu/ avp/collision_data/ (unpublished).

${ }^{53}$ A. V. Phelps, J. Phys. Chem. Ref. Data 20, 557 (1991).

${ }^{\mathbf{5 4}}$ A. V. Phelps, J. Appl. Phys. 76, 747 (1994).

${ }^{55}$ J. Schulze, E. Schüngel, Z. Donkó, and U. Czarnetzki, Plasma Sources Sci. Technol. 20, 015017 (2011)

${ }^{56}$ R. Kollath, in Encyclopedia of Physics, edited by S. Flügge (Springer, Berlin, 1956), Vol. XXI, p. 264.

${ }^{\mathbf{5 7}}$ B. Horváth, M. Daksha, I. Korolov, A. Derzsi, and J. Schulze, Plasma Sources Sci. Technol. 26, 124001 (2017).

${ }^{58}$ B. Horváth, J. Schulze, Z. Donkó, and A. Derzsi, J. Phys. D Appl. Phys. 51, 355204 (2018)

${ }^{\mathbf{5 9}}$ A. V. Phelps, L. C. Pitchford, C. Pédoussat, and Z. Donkó, Plasma Sources Sci. Technol. 8, B1-B2 (1999).

${ }^{60}$ T. Mussenbrock and R. P. Brinkmann, Appl. Phys. Lett. 88, 151503 (2006).

${ }^{61}$ U. Czarnetzki, T. Mussenbrock, and R. P. Brinkmann, Phys. Plasmas 13, 123503 (2006).

${ }^{62}$ T. Mussenbrock and R. P. Brinkmann, Plasma Sources Sci. Technol. 16, 377 (2007).

${ }^{63}$ T. Mussenbrock, R. P. Brinkmann, M. A. Lieberman, A. J. Lichtenberg, and E. Kawamura, Phys. Rev. Lett. 101, 085004 (2008).

${ }^{64}$ Z. Donkó, J. Schulze, and U. Czarnetzki, Appl. Phys. Lett. 94, 131501 (2009). 